\title{
On the Interdependence of Post-Error Control and Memory
}

\author{
Hannah Dames ${ }^{1,2,3} \&$ Christina U. Pfeuffer ${ }^{1}$ \\ ${ }^{1}$ Albert-Ludwigs-Universität Freiburg, Department of Psychology, Freiburg, Germany \\ ${ }^{2}$ Albert-Ludwigs-Universität Freiburg, Department of Computer Sciences, Freiburg, \\ Germany \\ ${ }^{3}$ University of Zurich, Department of Psychology, Zurich, Switzerland
}

\section{Author Note}

This research was supported by funding from the Der Verband der Freunde der Universität Freiburg e.V. (Experiment 1; Hannah Dames).

The data as well as experiment files and syntaxes are publicly available on OSF (https://osf.io/zvty6/). Correspondence should be addressed to Hannah Dames, E-mail: damesh@cs.uni-freiburg.de

(C) 2021, American Psychological Association. This paper is not the copy of record and may not exactly replicate the final, authoritative version of the article. Please do not copy or cite without authors' permission. The final article will be available, upon publication, via its DOI: 10.1037/xlm0001057 


\begin{abstract}
Post-error cognitive control processes are evident in, first, post-error slowing (PES) and posterror increased accuracy (PIA). A recent theory (Wessel, 2018) proposes that post-error control disrupts not only ongoing motor activity but also current task-set representations, suggesting an interdependence of post-error control and memory. In two experiments, we directly tested this interdependence using response repeat/switch targets. As participants' memory of the previouslygiven response determined their next response, we predicted that participants would not show post-error performance improvements, or may even exhibit decrements (memory obstruction hypothesis). In line with a weak version of the memory obstruction hypothesis, participants' performance did not improve post-error regardless of preparation time across several measures (intertrial interval $500 \mathrm{~ms}-3000 \mathrm{~ms}$ ). This could not alternatively be explained by a lack in posterror processing or error awareness. Thus, our results suggest an interdependence of memory and cognitive control in post-error processing and we offer a novel way of directly assessing this interdependence using response repeat/switch targets.
\end{abstract}

Keywords: Cognitive control, error processing, working memory, post-error slowing, post-error increased accuracy 


\section{Introduction}

Monitoring whether our behavior produces a desired outcome or fails to do so is essential to learn and flexibly adapt our actions to changing environmental conditions. For instance, after committing an error, post-error adaptions aim to improve future behavior (e.g., Danielmeier \& Ullsperger, 2011; Jentzsch \& Dudschig, 2009; Rabbitt, 1966; Wessel, 2018). A recent theory by Wessel (2018) proposes that post-error adaptations do not only stem from the inhibition of ongoing motor activity but also from the inhibition of the current task-set representation in (working) memory, that is an inhibitory cognitive control process (see Egner, 2008, for the idea that cognitive control in essence entails the strategic maintenance and adjustment of task-sets; see also e.g., Bugg \& Crump, 2012; Crump \& Logan, 2010; Dreisbach, 2012; Whitehead \& Egner, 2018). Here, we directly tested this theoretical assumption to examine interdependencies between memory and post-error cognitive control processes. Simultaneously, we accounted for the time available for error processing as well as interindividual differences in participants' error awareness that might have yielded additional influences.

After committing an error, humans slow down (e.g., Notebaert et al., 2009; Rabbitt, 1966). This prolonged reaction time (RT) in post-error trials is known as post-error slowing (PES; e.g., Notebaert et al., 2009; Rabbitt, 1966). With longer time intervals until the next response, attentional focusing towards task-relevant information is additionally observed in a post-error increase in accuracy (PIA; e.g., Maier et al., 2011).

\section{Wessel's Adaptive Orienting Theory of Error Processing}

Wessel (2018) provides a detailed explanation for why and when we observe post-error adaptations. He assumes that both PES and PIA reflect inherently adaptive post-error control processes (i.e., processes aimed at facilitating or improving future behavior $=$ cognitive control). 
Wessel (2018) proposes that unexpected events in general, not just errors (see e.g., Band et al., 2009, for evidence that the EEG signature of unexpected action outcomes resembles errors) trigger a two-step cascade. First, ongoing cognitive and motor representations are rapidly inhibited. In turn, this then facilitates a subsequent automatic attentional orienting towards the source of the expectancy violation (here the error), that is the unexpected mismatch between the intended and the actual action outcome. Only then, this automatic cascade enables error-specific controlled processing such as a perceptual retuning or task-set reconfigurations finally leading to behavioral improvement (see Wessel, 2018, for details).

Assessing post-error processing, Wessel (2018) distinguishes between processes that are automatic (i.e., not controlled processes; fast and not error-specific) and controlled (slow and error-specific). The exact contributions of automatic and controlled processes to PES and PIA effects have not been elucidated yet. Based on Wessel (2018), PES initially originates from automatic error processing, but PES effects arise when post-error processing (automatic and/or controlled) is prematurely terminated. That is, PES likely consists of both an automatic and a controlled component. PIA effects are observed only once controlled post-error processing, allowing for an increase in accuracy, has successfully terminated (see Wessel, 2018). This suggests that PIA effects mostly reflect controlled post-error processing.

\section{The Evolution of Post-Error Adaptations over Time}

According to Wessel (2018), rapid, automatic error-related processes (inhibition of ongoing cognitive and motor representations and attentional orienting towards the error) enable subsequent, slower, controlled error-related processes (e.g., task-set reconfiguration, perceptual tuning). Accordingly, post-error adaptations show an evolution over time (Danielmeier \& Ullsperger, 2011; Jentzsch \& Dudschig, 2009; van der Borght et al., 2016). This leads to clear 
predictions regarding the time frames following an error during which PES and PIA effects should be observed.

For PIA, those predictions are straightforward: Only after participants had sufficient time to finish controlled error-specific adaptations before their next action (i.e., for longer intertrial intervals, ITIs, i.e., longer response-stimulus intervals, RSIs), PIA is observed (Jentzsch \& Dudschig, 2009; Wessel, 2018). For such long ITIs, the existing task-set can be successfully reconfigured and tuned resulting in better accuracy (Wessel, 2018). In contrast, when a taskrelevant stimulus requires a response before this controlled post-error processing has finished (i.e., for short ITIs), instead of improving in task performance, accuracy does not improve (e.g., Buzzell et al., 2017) or may even drop (e.g., due to an attentional bottleneck caused by ongoing post-error adaptions; Jentzsch \& Dudschig, 2009).

PES has frequently been linked to an error-related orienting response which has in turn been associated with early reduced perceptual sensitivity, increased decisions bounds, and inhibitory control following errors (Buzzell et al., 2017; Ullsperger \& Danielmeier, 2016; MarcoPallares et al., 2008; Purcell \& Kiani, 2016). Wessel (2018) argues that as part of the rapid, automatic cascade following an error, attention is drawn away from the task-set. PES can be observed when a stimulus presentation requires a premature reorienting of attention, back towards the previously activated task-set before the source of an error has been identified (i.e., when the response target is presented before automatic error-related processing has finished) or before adaptive processing has finished (i.e., when the response target is presented before the controlled error-related processing has finished; see Wessel, 2018). PES then results from the delay associated with the reorienting of attention back toward the currently unattended and/or inhibited task-set. Consequently, PES originates during the early automatic cascade following an 
error but occurs both based on subsequent automatic and/or controlled post-error processing. When given sufficient time (i.e., long ITIs or RSIs) to complete post-error processing (e.g., reconfiguring the previously activated task-set and reinstating it in memory), PES reduces or vanishes (see e.g., Buzzell et al., 2017; Jentzsch \& Dudschig, 2009; Purcell \& Kiani, 2016; Ullsperger \& Danielmeier, 2016; van der Borght et al., 2016; but see Wessel, 2018 for how PES can also result from a strategic speed-accuracy trade off ${ }^{1}$ ).

\section{The Role of Task-Set Representations in Wessel's Theory}

A key aspect of Wessel's (2018) theory is the assumption that the rapid inhibition following errors does not only affect motor processes, but also cognitive representations such as the current task-set (i.e., the representation of the task goal, task-relevant stimuli and responses as well as their mappings; see e.g., Kiesel et al., 2010; see Künzell et al., 2018, for a recent discussion of what a task entails), which allows us to goal-directedly act according to task requirements. Wessel (2018) proposes that the inhibition of the current task-set representation helps to draw attention away from the current task-set representation toward the source of an

${ }^{1}$ Wessel (2018) argues that there are the two types of PES, namely, orienting-related PES and strategic PES. In the present work we focus on orienting-related PES which occurs when adaptive processing must prematurely stop (as described above). Note, however, that strategic PES can be observed when it is strategically beneficial to slow down after an error, for instance, when a premature response was the source of an error (see Wessel, 2018). We will briefly refer to potential influences of strategic PES in the present study in the Discussion of Experiment 1. 
error. The inhibition of the current task-set thus frees up resources and supports quick subsequent, controlled shifts of attention.

The assumption that during the proposed automatic cascade following an error, the current task-set representation is inhibited, has several interesting implications. It implies that early, automatic error processing disrupts (and/or inhibits) task-set representations that are currently held in an active state (e.g., in working memory). At this time, those task-set representations should be less accessible. Yet, during subsequent controlled error-specific adaptations, the task-set can be reconfigured and reinstated to enhance subsequent performance. This means that memory of the task-set that "survives" the initial disruption during the automatic cascade following an error, is essential to drive such efficient cognitive control processes. This suggests a strong interdependence of task-set memory and error-specific cognitive control processes. To assess this interdependence, here we pitted post-error inhibitory control processes against memory retrieval of current task-set related representations to create boundary conditions that allowed us to study how memory and post-error cognitive control processes are intertwined.

\section{Does Post-Error Cognitive Control Obstruct Task-Set Representations?}

So far, to the best of our knowledge, the only evidence for Wessel et al.'s (2018) idea that errors inhibit task-set representations in memory comes from a study that addressed the topic rather indirectly (Wessel et al., 2016). In this study, unexpected sounds were presented during the memory maintenance period in a verbal working memory task (WM). The authors found that these unexpected sounds led to deficits in WM resulting in lower accuracy on subsequent probes as compared to non-surprising events. In particular, the surprising tones were associated with a disruption of ongoing WM processes via the same fronto-basal ganglia mechanism (i.e., the same independent neural signal component) that is also recruited when stopping actions in the stop- 
signal task (Wessel, 2018). Wessel et al. used the recruitment of this neural suppressive mechanism underlying the stopping of motor actions to explain the decreased WM performance following surprising events. They concluded that unexpected events disrupt not only motor processes, but also ongoing WM processes.

During correct responding and prior to an error the current task-set is maintained in an active state in WM (for the idea that task-set representations may be maintained in WM in a reduced or compressed state via cues to long-term memory, LTM, see Kikumoto \& Mayr, 2017). WM is essential to human cognition as it holds mental representations temporarily available for use in thought and action (Oberauer et al., 2018). Other than LTM, WM is assumed to be of limited capacity as shown in various WM tests, where participants' performance declines as the number of elements to-be-remembered, the set size, increases (e.g., Cortis et al., 2015; Woods et al., 2016). Because WM capacity is limited, its content needs to be constantly updated to focus on currently relevant information. Given Wessel et al.'s (2016) finding regarding decreased WM performance following surprising events, the question arises whether post-error processing additionally taxes WM thus affecting other WM content.

Although Wessel et al.'s (2016) results suggest that WM is affected by some form of suppression or inhibition (see Wessel, 2018), the cognitive mechanisms underlying these findings are still unclear. That is, how post-error cognitive control and (working) memory interact has not been unraveled yet. For instance, it is unclear whether WM disruptions are accompanied by the active removal of information from WM or whether information in WM is inhibited or simply not rehearsed any more. Irrespective of the degree to which WM processes are obstructed by error-related processing, we assumed that following an error, current task-set related representations maintained in WM are disrupted (or removed from maintenance/rehearsal and 
thus dropped). We therefore set out to further examine post-error task-set related memory to better understand the interdependence of memory and cognitive control in post-error processing.

\section{The Present Research}

In typical speeded-response paradigms, an interruption of the current task-set related representations in WM would have no dramatic influence on participants' performance. That is, the repetitive nature of the majority of experimental tasks (e.g., consistently pressing a specific key in response to a target) also results in the formation of LTM representations of these stimulus-response (S-R) mappings (e.g., Mayr et al., 2014; Waszak et al., 2003). Hence, a reorientation towards the current task-set can easily be accomplished by re-activating these highly familiar S-R rules in WM via cues to LTM. To test the interdependence of post-error inhibitory control processes and (working) memory, we therefore, designed an experimental setting in which the task-set related representations need to be flexibly updated based on the previous trial's response as indicated by a response repeat/switch target. That is, we created a setting in which the retrieval of S-R rules from LTM by itself was insufficient for successful responding.

In tasks using response repeat/switch targets, participants' memory of the previously given response is essential to determine how to respond in the following trial on the basis of the response repeat/switch target. For instance, when the participants pressed the left key in trial ${ }_{\mathrm{n}-1}$, a response repeat target requires again a left key press and a response switch target requires participants to press the right key. Thus, the to-be-executed response needs to be constantly 
updated based on the prior response ${ }^{2}$. It is also likely that participants proactively (before the onset of the following target) prepare and maintain all four possible mappings (i.e., prior right/left + response repeat target $=$ right/left, prior right/left + response switch target $=$ left $/$ right $)$ or at least the two possible target-based mappings for the just given response (e.g., prior right: response repeat target $=$ right, response switch target $=$ left). In addition with participants' memory for the previous response, such task-set translations would then need to be maintained and updated (based on the previous response) in WM. Interrupting this updating process inbetween trials and simultaneously withdrawing attention from these rather fragile current task-set related representations in the process of post-error adaptations (possibly even fully removing corresponding content from WM), would make it much more difficult if not impossible to reconfigure the task-set related information (i.e., the construed task-set taking the prior response

${ }^{2}$ Please note that in the present study, the task-set (the S-R rules) consisted of the corresponding vowel-repeat/consonant-switch mappings (or vice versa depending on the participant's assignment) which had to be represented in LTM. Here we assume that in WM (as the activated part of LTM; see Cowan, 1999; Oberauer, 2002, 2009), memory for the previous response was used in combination with activated task-set representations in LTM to determine a correct response in trialn. In addition, we assumed that proactively prepared task-set translations developed before the following trial's target appears can also be maintained in WM (see the following description). For the sake of brevity, in the following we subsume all of these representations in WM as task-set related representations in WM. 
into account) when post-error processes are completed. We will refer to this idea as the memory obstruction hypothesis.

To address the interdependence of (working) memory and post-error cognitive control (i.e., to test the memory obstruction hypothesis), in two experiments, participant's to-beperformed response in a given trial $\mathrm{n}_{\mathrm{n}}$ either repeated or switched as compared to the correct response of the preceding trial $\mathrm{n}_{\mathrm{n}-1}$. Importantly, the correct response always served as a reference for the subsequent response repeat/switch target. For instance, when committing an error using a left response, a following response repeat target required a right response as this would have been the correct response of the preceding trial $\mathrm{n}_{\mathrm{n}-1}$. Thus, to respond correctly, participants needed to remember both the target-repeat/switch instruction mapping and their previous response to update the existing task-set translation accordingly and perform above chance. Additionally, when they committed an error, participants needed to become aware of their errors and to use the appropriate reference, the correct response of the previous trial, to successfully update the required response based on the response repeat/switch target.

In this design, we expected that particularly the task-set reconfiguration as part of the controlled post-error processes should be affected when memories for the past responses and/or task-set related representations are obstructed. That is, according to the memory obstruction hypothesis, we first predicted that task-set related representations (task-set translation and/or past responses) should be less/not accessible in post-error trials as compared to post-correct trials. As a consequence, the controlled post-error adaptions should fail or be prolonged resulting in two observations: First, PES should occur for a prolonged time (i.e., also for long ITIs), because controlled post-error processes cannot finish even given ample time. Second, PIA should not be observed for the exact same reasons: As controlled post-error processing cannot finish even 
when ample time is given, PIA effects building on the results of controlled post-error processing should not emerge.

\section{Strong Version of the Memory Obstruction Hypothesis}

A strong interpretation of the memory obstruction hypothesis states that the task-set inhibition associated with post-error processing is accompanied with the rapid and complete removal of the currently activated task-set representations (e.g., memory for the prior response and task-set translations) from WM (see Lewis-Peacock et al., 2018 and Oberauer, 2018, for the idea that information can be removed from WM). In that case, to respond correctly in post-error trials, participants need to fully rely on LTM to reinstate the task-set. We assumed that the usage of response repeat/switch targets would create a situation in which errors prevent participants from accessing task-set related representations in WM when, at the same time, LTM representations are of limited help in reinstating the prior, performance-based task-set.

Wessel (2018) suggests that, if a response is required while the automatic cascade of inhibition-reorienting has not finished yet and the current task-set is still suppressed, two observations should be made: First, the reinstantiation of the original task-set will take additional time, resulting in an orienting related PES when both the automatic as well as the controlled processes are not completed yet. Second, if the task-set is still inhibited when following responses are required, accuracy should be close to chance level. Here, we extend this line of reasoning to the prediction of the strong version of the memory obstruction hypothesis: Using response repeat/switch targets, accuracy in post-error trials should be close to chance or at least drastically lower than in post-correct trials. 


\section{Weak Version of the Memory Obstruction Hypothesis}

A weaker version of the memory obstruction hypothesis states that task-set related representations (e.g., task-set translations and/or memory for past responses) in WM are not fully removed from WM but are rather not (or less) attended (not actively maintained) while post-error processing is ongoing. Accordingly, participants may still reinstate the task-set even when confronted with flexibly-shifting task-set translations. Nevertheless, participants' response accuracy in post-error trials should be lower than in post-correct trials. That is, depending on the degree of memory obstruction one assumes, we would expect at least evidence against a PIA effect or, in stark contrast to prior studies on post-error processing, even an inversed PIA effect.

Even if participants could still partially draw on LTM representations, tactile aftersensations of prior responses, or supporting strategies like lifting the finger they just responded with (strategies likely to be applied on both response repeat and switch target trials to a similar degree as the nature of the upcoming target was unpredictable), performance should still decrease in post-error trials as compared to post-correct trials. As discussed, one could also assume that participants proactively prepare and maintain task-set translations. Nevertheless, these mappings would then need to be maintained in WM. Thus, even under these conditions, the memory obstruction hypothesis would predict a disruption of such maintenance processes and/or a (partial) removal of these proactive task-set translations from WM. Thus, even considering options for proactive preparation, we should observe post-error performance costs, that is no or an inversed PIA effect.

Although the memory obstruction hypothesis is derived from Wessel's theory (2018), the theory itself does not clearly favor the strong or the weak version of our memory obstruction hypothesis. The strong version extends Wessel's hypothesis that task-set representations are 
inhibited shortly following an error by assuming that memory is obstructed in a way that taskrelevant information is removed from WM. If memories for the previously given response or task-set translations are lost in that process (as LTM does not suffice to reactivate that information), performance should be at chance level post-error. A weak version of the memory obstruction hypothesis assumes that this information is not completely lost, but reactivation of this information should nevertheless be difficult post-error in the present paradigm and thus, predicts no or an inversed PIA effect (i.e., no post-error improvement). In contrast to the strong version, however, the weak version of the memory obstruction hypothesis does not predict posterror accuracy to drop to chance level. Both hypotheses are in line with Wessel's (2018) predictions and only an empirical assessment can constitute further support for one over the other.

\section{The role of the Intertrial-Interval and Target Type}

Importantly, the memory obstruction hypothesis also makes clear predictions regarding the impact of ITI/RSI duration on PIA effects. Contrary to prior research on post-error adaptations (e.g., Buzzell et al., 2017; Jentzsch \& Dudschig, 2009), we expected to observe no PIA effect or even an inversed PIA effect regardless of ITI duration (i.e., preparation time). When memory for the task-set translation and past responses is obstructed in trials following an error, post-error processing cannot finish even when ample time is given. Thus, when required task-set translations vary on a trial-by-trial level, even at long ITIs, additional preparation time should hardly help to recover the disrupted task-set translations. Note that prior studies mostly used shorter ITI/RSI durations (e.g., Buzzell et al., 2017; Jentzsch \& Dudschig, 2009). We intently chose comparably long durations for both our short and long ITI/RSI, as chances of 
observing a PIA effect increase with preparation time. Thus, long ITI/RSI durations represent the more critical test conditions for the absence/inversion of PIA effects.

Moreover, based on the memory obstruction hypothesis, costs of inhibiting the current task-set related representations post-error should also be stronger in response switch than in response repeat trials. In post-correct trials, participants need to retrieve the reference response (step 1) and, additionally, invert the previous response when a response switch target is presented (step 2). In post-error trials, for both response switch and repeat targets, participants need to correct their previous response (step 0: adapt the reference response; e.g., left key press was incorrect; right key press would have been correct and is the reference for the next trial). As participants do not already know what the next trials target will be, we suggest that this correction of the reference response constitutes an initial step (step 0 ) that is necessary after an error but not after a correct response. That is, we presume that participants first set the reference response before encountering the next target. Thus, both following correct responses and errors, a response switch target requires participants to retrieve the reference response (step 1) and then devote cognitive resources to invert it and execute the opposite response (step 2). However, posterror, additional capacities are required for error-related processing. In sum, response switch targets should further tax participants after an error (post-error: step 0-2 + error-related processing; post-correct: step $1+2$ ) even though the same response is required when a response switch target follows an error. As a result, the recruitment of participants' limited processing resources by the post-error cognitive control processes should make it more difficult for participants to respond correctly to response switch targets as compared to response repeat targets. This should especially be the case in post-error as compared to post-correct trials when post-error processing requires additional resources. Thus, we should not only observe an effect of 
target type, but also an interaction of PIA effects and target type with even further reduced/inversed PIA effects for response switch targets.

The memory obstruction hypothesis is based on the assumption that motor and cognitive representations are inhibited following an error. Accordingly, we also expected to find a pronounced PES effect in our design. However, contrary to prior studies (Buzzell et al., 2017; Jentzsch \& Dudschig, 2009; Purcell \& Kiani, 2016; van der Borght et al., 2016), we assumed to find PES for both short and long ITIs as the reinstatement of the task-set related representations should fail or take substantial time in the current study using response repeat/switch targets. Consequently, participants should have greater difficulties to select a response in post-error as compared to post-correct trials even for long ITIs. In parallel with the reasoning for the inverse PIA effect, PES should consequently also be greater for response switch than for response repeat targets in post-error trials and even more so than in post-correct trials.

\section{Manipulation Checks: PES and The Role of Error Awareness in Post-Error Control}

To ensure that in our response repeat/switch target paradigm participants engaged in posterror processing following an error in a way comparable to prior studies (for an overview see Danielmeier \& Ullsperger, 2011), we conducted two manipulation checks. First, if participants enter the automatic processing cascade following an error (Wessel, 2018), they should slow down relative to post-correct trials at least for short ITIs irrespective of our other results. That is, if we had not found a PES for short ITIs, we could not have interpreted our results in light of Wessel's theory and prior research on post-error processing. In that case, we would have had reason to believe that post-error processing in our paradigm might have generally differed from previously used paradigms, making results incomparable and thus inconclusive. 
Second, Wessel's (2018) theory on error processing recognizes the relationship between error awareness and post-error adaptations. In this respect, it has to be noted that the orienting response plays an important role in generating conscious error-awareness (Ullsperger et al., 2010; Wessel, 2012). Wessel (2018) argues that error awareness should be proportional to the magnitude of the automatically generated orienting response. As a result, controlled adaptations following the automatic cascade should be proportional to the degree of error awareness. In spite of some evidence indicating that not consciously perceived errors do not result in PES (e.g., Nieuwenhuis et al., 2001; Wessel et al., 2011), there is a lack of studies investigating interindividual differences in the ability to detect errors and the discussed post-error adaptions. We conceptualized the awareness of one's response accuracy (response accuracy awareness, short RAA) as a decision process in which the available evidence that a correct or incorrect response (also error awareness) was given accumulates until a decision criterion is either reached (see Steinhauser \& Yeung, 2010) or the evidence accumulation process is aborted. We measured RAA with two goals: First, assuming a potential influence of error awareness, we needed to ensure that participants became aware of committing an error in the current design to a degree comparable to prior studies (e.g., Grützmann et al., 2014; Hughes \& Yeung, 2011; Murphy et al., 2012; Shalgi et al., 2009; Shalgi \& Deouell, 2012; Wang et al., 2020). If participants had not reached sufficient RAA, our results could have been alternatively explained by the increased difficulty of the current paradigm that prevented participants from developing error awareness. Second, we assumed that a person's error awareness is related to the strength of their individual error-related monitoring processes. That is, individual error awareness might partly account for individual variations in the post-error control processes we assessed. We thus assessed and controlled for participants' error awareness. 


\section{Experiment 1}

The goal of Experiment 1 was to test the memory obstruction hypothesis. First, we examined whether a disruption of task-set related representations indeed resulted in an inversed PIA effect, whereas robust PES was still observed (at least for short ITIs). Furthermore, if the memory obstruction hypothesis holds true, both the inversed PIA effect and PES should be stronger in response switch as compared to response repetitions trial. However, for both, we did not expect an influence of ITI duration. Finally, we assessed error awareness to control for it and investigate its potential impact on post-error adaptations and related memory processes.

\section{Method}

\section{Participants and Exclusion Criteria}

Twenty-nine participants took part in the experiment for course credit. Buzzel et al., (2017) collected data from 23 participants and had sufficient power to measure behavioral effects of interest for our analyses (e.g., that PIA is reduced for short as compared to long ITIs with an effect size of $d=1.07)$. Thus, we aimed to have data from at least 24 participants for statistical analyses after applying our exclusion criteria. All participants were naive to the purpose of the experiment and had normal or corrected-to-normal vision. Three participants had to be excluded due to either an early abortion of the experiment $(n=2)$ or because they did not use the left and right index fingers to respond during the task as they were instructed $(n=1)$. Furthermore, posterror adaptation effects change when errors occur frequently (see e.g., Notebaert et al., 2009), we therefore excluded data from participants with an overall error rate of $50 \%$ or more $(n=1)$. In addition, as analysis of post-error adaptations requires a sufficient number of erroneous responses, we discarded the data of another participant who did not have observations in each cell. In sum, the data of five participants were discarded, resulting in a sample of 24 participants 
for analyses $\left(M_{\text {age }}=22.33, S D=4.14\right.$, range $19-33$ years; $91.7 \%$ female, $8.3 \%$ male $)$. The present study was conducted in adherence to the Declaration of Helsinki and the guidelines set by the local ethics committee.

\section{Stimuli and Apparatus}

The experiment took place in a sound attenuated laboratory room. Participants sat approximately $60 \mathrm{~cm}$ from a 24" (1920x1080) LCD monitor. The experiment was controlled via the experiment software E-Prime ${ }^{\circledR}$ version 2.0.10.356 (Psychological Software Tools Inc., Sharpsburg, PA, USA). Behavioral responses were collected via key presses on external response keys (inter-key distance: $13.5 \mathrm{~cm}$ ). Participants placed their left/right index fingers on the left/right key, respectively. The screen background was black throughout the experiment.

\section{Procedure}

The experiment consisted of one practice block of 51 trials and ten experimental blocks of 51 trials. Only in the first trial of a block, the target was a left-/right-pointing arrow and participants responded accordingly to set an initial response. Thus, there were 500 experimental trials in total (excluding the first trial of each block). There were two types of blocks: In the test blocks, participants' responses triggered a feedback that was informative about their response accuracy (6 of 10 blocks). In the response accuracy awareness (RAA)- blocks, on a proportion of trials, participants first indicated their belief about the accuracy of their responses before receiving feedback (4 of 10 blocks). Per half of the experiment, participants first completed three test blocks and then two RAA blocks. In addition, the duration of the intertrial interval (ITI) varied between the first and the second half of the experiment. Whether the experiment started with a short $(500 \mathrm{~ms})$ or long $(1700 \mathrm{~ms})$ ITI duration was counterbalanced across participants. The ITI of $500 \mathrm{~ms}$ equals a RSI of $1300 \mathrm{~ms}$ (300 ms response-feedback interval $+500 \mathrm{~ms}$ 
feeback $+500 \mathrm{~ms}$ ITI) and the ITI of $1700 \mathrm{~ms}$ equals a RSI of $2500 \mathrm{~ms}$. Note, that previous research used mostly used shorter ITI/RSI durations (e.g., Jentzsch \& Dudschig, 2009: 50/100 ms vs. $1000 \mathrm{~ms}$ ). As chances of observing PIA effects are higher for longer ITIs, we chose comparably long durations for both our short and long ITI. These long ITIs represent the more conservative test of our hypothesis that even when participants are given ample preparation time, they cannot improve in trials following an error due to memory obstruction. Each trial started with an ITI during which a white fixation cross was presented centrally (see Figure 1 for the trial structure). Next, the target $\left(100 \mathrm{~ms}, 0.7^{\circ}\right.$, white $)$ - a vowel (A, E, I, O, U) or a consonant (R, F, T, Q, V) - indicated whether the to-be-performed response was the same response (response repeat target) or the opposite response (response switch target) as compared to the correct response of the preceding trial. Participants were instructed that the correct response of the previous trial served as the reference for the subsequent response repeat/switch target. ${ }^{3}$ The vowel/consonant-response mapping was counterbalanced across participants and targets

${ }^{3}$ In addition, participants were told that in case of an error, the correct response for the response repeat/switch target referred to the response that would have been correct in that the previous trial. That is, participants were instructed that, upon an error, the response they did not perform served as a reference and that they should adapt their response accordingly (German original instruction: "Dies bezieht sich immer auf die Taste, die du zuvor hättest drücken sollen. Falls du im vorherigen Durchgang einen Fehler gemacht hast, musst du deine Reaktion also entsprechend anpassen. Die korrekte Reaktion im vorherigen Durchgang wäre dann die Taste gewesen, die du NICHT gedrückt hast”). 
appeared equally frequently per block. After the target disappeared, participants had another 700 $\mathrm{ms}$ to respond (response deadline: $800 \mathrm{~ms}$ ). Then text feedback was presented $(500 \mathrm{~ms}$, red) for premature (“'Zu früh!”/ "Too early!', accidental responses given before target onset) or omitted responses (“Zu langsam!"/ "Too slow!", trials on which no response was given within the response deadline). On all other trials, participants' responses triggered a visual feedback (coloured circle) in the center of the screen that indicated whether the response was correct or not $\left(\right.$ correct $=$ green circle, incorrect $=$ red circle, $\left.500 \mathrm{~ms}, 1.3^{\circ}\right)$ 


\section{Figure 1}

Illustration of the Trial Sequence in Experiments 1and 2

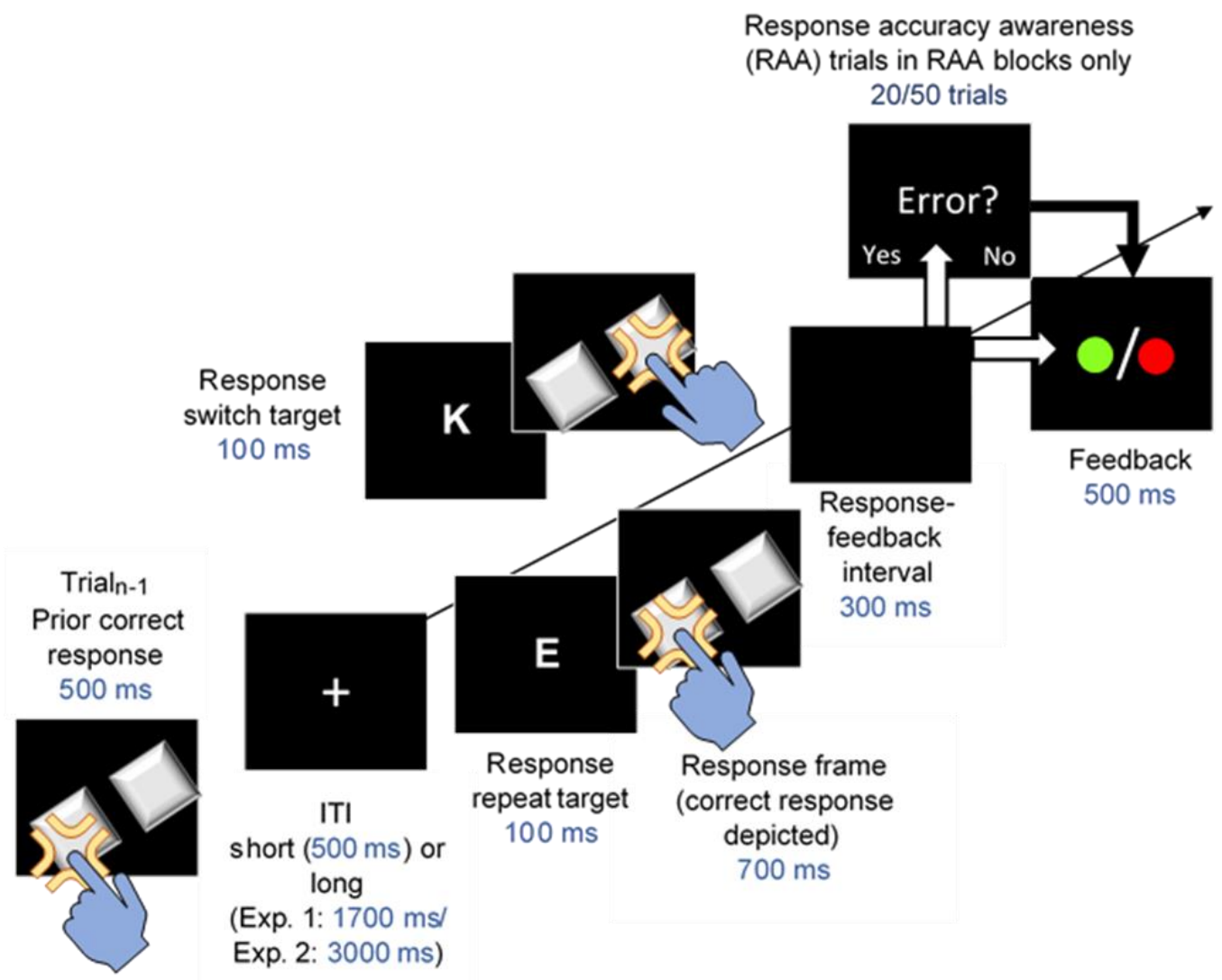

Note. A correct, left response is given in trial $\mathrm{n}_{\mathrm{n}-1}$. In trial $\mathrm{l}_{\mathrm{n}}$, the fixation cross presented during the intertrial interval (ITI, $500 \mathrm{~ms}$ vs. Experiment 1: $1700 \mathrm{~ms} /$ Experiment 2: $3000 \mathrm{~ms}$ ) was substituted by the forced-choice response repeat or switch target $(100 \mathrm{~ms})$. The target was followed by a blank screen $(500 \mathrm{~ms})$ during which participants could still respond (response deadline $800 \mathrm{~ms}$ ). Participants' responses (example showing correct case) triggered feedback in the form of a green or red circle located in the center of the screen (green = correct; red = incorrect) which appeared after a response-feedback interval of $300 \mathrm{~ms}$. In total, the RSI thus summed up to $1300 \mathrm{~ms}$ in the short ITI condition and $2500 \mathrm{~ms}$ (Exp. 1)/ $3800 \mathrm{~ms}$ (Exp. 2) in the long ITI condition. In 20/50 trials of the response accuracy awareness (RAA) blocks (4/10; randomly intermixed in these blocks) participants did not receive direct feedback. Instead, they first indicated whether their given response was correct or incorrect by responding to either "Richtig?" (for "correct?") or "Fehler?" (for “error?”; randomly chosen) by selecting "Ja" (for "Yes") or "Nein" (for "No"; chosen randomly each time). For easier international interpretability, the figure presents the English translation. 
In test blocks, feedback followed participants' responses after a brief response-feedback interval of 300 ms. In RAA blocks, to assess participants's awareness of the accuracy of their responses, in 20 trials per block (RAA trials; randomly intermixed in these blocks) participants were first asked to state whether they thought their just given response was correct or incorrect. This was done by presenting participants either with the question "Richtig?" ("correct?") or "Fehler?" ("error?"; question randomly chosen per trial and equally frequent) in white in the middle of the screen. At the same time, two response options, "Ja" ("Yes") and "Nein" ("No"; spatial location chosen randomly each time and equally frequent) were presented on the left and right underneath the question. Participants were instructed to respond by pressing the key corresponding to the position of their answer. After their response and the response-feedback interval, the feedback was presented.

Participants were instructed to respond as fast and accurately as possible. Furthermore, they were instructed to do their best when judging the correctness of their responses and to respond according to their intuition when they were unsure.

\section{Data Analysis}

We used a mixed models approach (for an overview see Baayen et al., 2008; Judd et al., 2012). RTs (PES) analyses were run using linear mixed models (LMMs) and we analyzed the correctness of responses (PIA) using generalized mixed models (GLMMs) with a binomial link function. The (G)LMMs were analyzed using mainly the $R$ packages $l m e 4$ (Bates et al., 2015) and afex (Singmann et al., 2015). We obtained model predictions and graphics with the support of the ggeffects package (Lüdecke, 2018), and emmeans (Lenth et al., 2018).

For the LMM on participants' RTs, we used maximum likelihood (ML) estimation for model selection and restricted maximum likelihood (REML) estimation for slope estimates. $P$ - 
values for effects were obtained using the Kenward-Roger (Kenward \& Roger, 1997) approximation for denominator degrees of freedom. In order to quantify post-error slowing (PES), we differentiated between trials following incorrect (post-error trials) and correct responses (post-correct trials) as indicated by the factor response-correctness $n-1$.

Note, that there is also an alternative approach that can be taken to calculate PES. This approach takes only correct trials directly preceding an error, but not simultaneously following an error, into account for the assessment of post-correct trials. Although this approach has been discussed to be more reliable for PES analysis being immune to global performance fluctuations (Dutilh et al., 2012), it may be inflated by pre-error speeding. In addition, a great amount of data is lost. We note that the different versions to calculate PES are currently controversially discussed and have not been standardized yet (Schroder et al., 2020). We thus quantify PES differentiating between trials directly following incorrect (post-error trials) and all trials following correct responses (post-correct trials) as we deemed it more important not to overestimate the PES effect in our design. The resulting full model that converged properly (when removing the correlation among by-participant random effect terms) included a random intercept for participants and random slopes varying across participants for responsecorrectness $_{n-1}$, ITI, target type, and random slopes for all corresponding two-way interactions.

RTs for correct responses only were predicted using the fixed factors ITI duration $(1=$ short/500 ms vs. $-1=$ long/1700 ms $)$, response-correctness $n-1$ ( 1 = post-error, $-1=$ post-correct $)$, and target type $(1=$ repeat vs. $-1=$ switch $)$, and all corresponding interactions between these three factors. The block number was added as a covariate to account for changes over time. Furthermore, the model included participants' error awareness (the relative frequency of correctly stated errors over all RAA blocks per participant) as a continuous predictor. In order to 
analyze the impact of participants' error awareness on the strength of the PES effect, we also included an interactional term between error awareness and response-correctness $s_{n-1}$. The block number was added as covariate to capture effects due to fatigue or learning. We implemented the maximal random-effects structure justified by the design (as suggested by Barr et al., 2013; including by-participant random slopes for ITI duration, response-correctness ${ }_{n-1}$, target type, and their interactions). Yet, as the model including random correlated intercepts and slopes did not adequately converge, the correlations among random slopes were removed. In case the model did not converge, we would have first removed random slopes for higher level interactions of random effects, one by one starting with the random slope for the three-way interaction, then the random slope for the interaction between ITI and target type, then between responsecorrectness $_{n-1}$ and target type, then response-correctness $s_{n-1}$ and ITI. Next, we would have removed the random slope for target type, then ITI, and last response-correctness ${ }_{n-1}$. In between each step, we would have also removed random effects correlations. The final LMM that converged included a random intercept for participants and six random slopes varying across participants for response-correctness $\mathrm{n}_{\mathrm{n}-1}$, ITI, target type, and the interaction between target type and response-correctness $\mathrm{n}_{\mathrm{n}-1}$, the interaction between response-correctness $\mathrm{s}_{n-1}$ and ITI, and the interaction between ITI and target type excluding correlations among random effects.

For PIA analysis, GLMM estimates were computed with ML and binomial link functions using the "BOBYQA" optimizer with 1,000,000 iterations. The $p$-values were obtained via Likelihood Ratio Tests (LRT) and we additionally calculated the odds ratio statistic for interpretation of effect sizes (OR; see Szumilas, 2010). PIA was assessed by predicting the correctness of responses (incorrect response $=1$, correct response $=0$ ) using the same predictors (same fixed effects) as for the model on RTs. Following the approach taken for the PES analysis, 
the model also included participants' error awareness and the corresponding interaction with response-correctness $s_{n-1}$ as continuous predictors to assess the impact of error awareness on the strength of the PIA effect. The block number was added as covariate to capture effects due to fatigue or learning. Similar to the RT model, we started with the maximal random-effects structure justified by the design and reduced it the same way as we did for RTs until the model converged. The final GLMM that converged included a random intercept for participants and four random slopes varying across participants for response-correctness $\mathrm{n}_{\mathrm{n}-1}$, ITI, target type, and the interaction between target type and response-correctness $\mathrm{n}_{\mathrm{n}-1}$ excluding correlations among random effects.

Effect coding was used for all dichotomous fixed effects. All continuous measures were z-standardized. For each model, we calculated marginal and conditional $R^{2}$ statistics, based on Nakagawa et al. (2017). The marginal $R^{2}$ considers only the variance of the fixed effects, while the conditional $R^{2}$ takes both the fixed and random effects into account. For the analysis of posterror adaptations only test blocks were used to ensure that the interdispersed RAA questions could not influence the pattern of results (see Grützmann et al., 2014).

\section{Results}

The overall error rate in test blocks was $13.1 \%(S D=7.3 \%)$. Error rates in RAA blocks were higher $(18.9 \%, S D=9.1 \%)$ than in test-blocks, $t(23)=4.54, p<.001, d=0.70$. Figure $2 \mathrm{~A}$ shows the boxplots of participants' error rates for RAA-blocks and test-blocks. There were no participants with exceptionally high (> 3 SDs) error rates. Trials with response omissions $(n=279,2.3 \%)$ and premature $(n=18,0.2 \%)$ responses as well as trials preceding and following these trials (omitted: $n=507,4.2 \%$, premature: $n=33,0.3 \%$ ) were excluded from all analyses. 


\section{Figure 2}

Errors Rates and Response Accuracy Awareness in Experiment 1

A

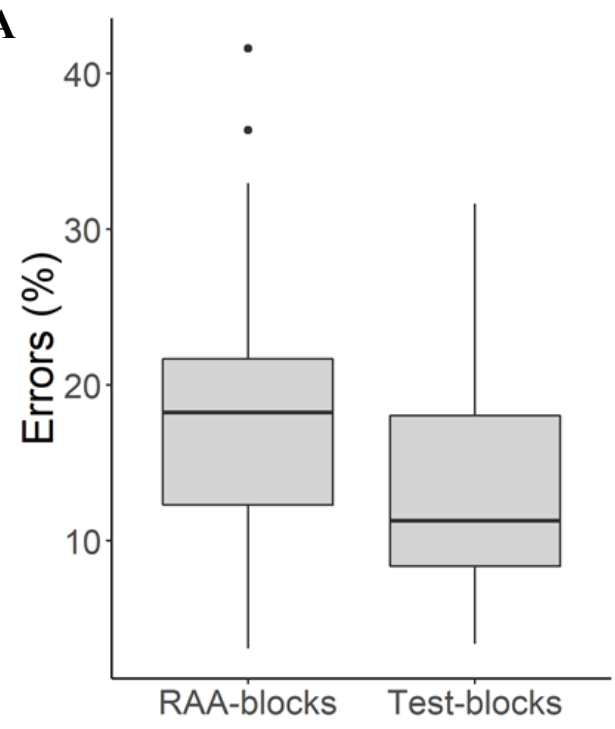

B

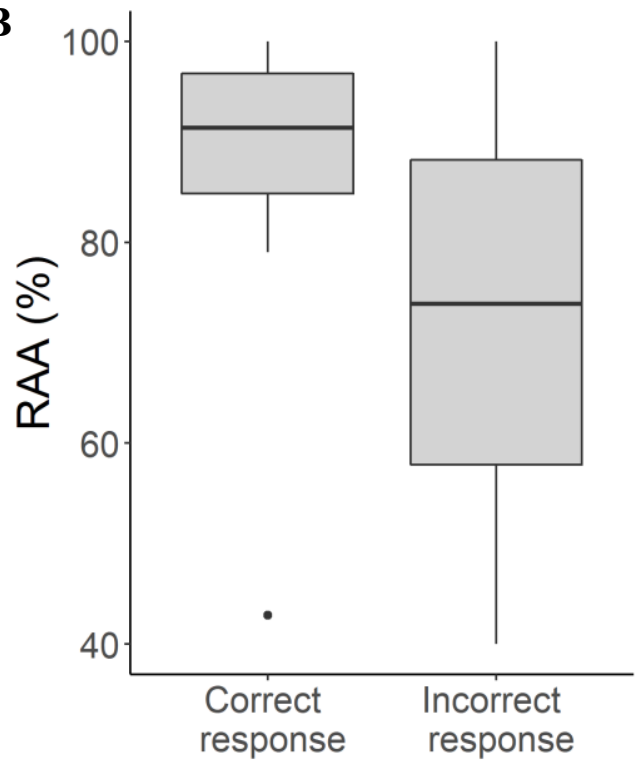

Note. A) Percentage of errors per participant displayed separately for RAA-blocks with response accuracy awareness (RAA) questions and test blocks without RAA-questions.

B) Response accuracy awareness (RAA) for correct and incorrect responses.

\section{Response Accuracy Awareness (RAA)}

Evaluation of participants' RAA- questions showed that erroneous responses were consistently rated as incorrect $(M=72.9 \%, S D=18.7 \% ; M d n=73.9 \%)$ and correct responses as correct $(M=89.2 \%, S D=12.1 ; M d n=91.4 \%)$ above chance $(50 \%)$ as illustrated in Figure $2 \mathrm{~B}$. For RAA in incorrect responses, no deviation from normality (Shapiro-Wilk test) was detected and a one-sample $t$-test revealed that the answers to the RAA questions were significantly above chance level $(50 \%)$ for incorrect responses, $t(23)=6.01, p<.001, d=1.23$. For correct 
responses, there was a slight deviation from normality. Thus, a Wilcoxon signed rank test was used also suggesting a response awareness above chance $(V=299, p<.001)$ for correct responses. Furthermore, RAA was significantly higher for correct than for incorrect responses (paired Wilcoxon signed rank test; $V=250, p<.001$, with a Median difference of $16.1 \%$ in RAA). The slight deviation from normality in RAA for correct responses should not affect PES or PIA, and therefore the respective participant was not excluded from the other analyses.

\section{Post-Error Slowing and Post-Error Increased Accuracy}

Mean RTs and error rates are displayed in Table 1. For the analysis of PES and PIA only trials 3-51 per block from test blocks were considered. Note that the first two trials of each block were excluded as the first trial only set the first response and the subsequent trial was thus not following a typical correct or incorrect response. For analyses of PES, error sequences including trials preceding and directly following such error sequences $(n=640,9.3 \%)$ were excluded. Of course, for the analysis of PIA, the first two repeating errors were kept and only the following trials of the error sequence and the trial following the end of the sequence were deleted $(n=133$, $1.1 \%)$. Furthermore, for both analyses of PES, to ensure a reliable measure of post-error adaptations, we did not consider trials which were in-between errors and thus rated as both a post- and pre-error trial $(n=56,0.5 \%)$. For PES analyses only correct responses were considered. Additionally, for PES analysis, trials with RTs deviating more than 3 standard deviations (SDs) from a participant's mean RT in the respective cell (PES: $n=33,0.3 \%$ ) were excluded. 
Table 1

Mean Reaction Times and Error Rates for Correct and Incorrect Trials in Experiments 1 and 2

\begin{tabular}{|c|c|c|c|c|c|}
\hline & & \multicolumn{4}{|c|}{ Response Target } \\
\hline & & \multicolumn{2}{|c|}{ Repeat } & \multicolumn{2}{|c|}{ Switch } \\
\hline & & \multicolumn{2}{|c|}{ Response Correctness $_{n-1}$} & \multicolumn{2}{|c|}{ Response Correctness $_{n-1}$} \\
\hline & & Correct & Incorrect & Correct & Incorrect \\
\hline \multicolumn{6}{|c|}{ PES: RTs (ms) } \\
\hline Exp. 1 & $\begin{array}{l}\text { Short ITI } \\
(500 \mathrm{~ms}) \\
\text { Long ITI } \\
(1700 \mathrm{~ms})\end{array}$ & $\begin{array}{l}460(33), \\
n=1178 \\
480(34), \\
n=1336\end{array}$ & $\begin{array}{l}490(56), \\
n=140 \\
497(51), \\
n=104\end{array}$ & $\begin{array}{l}461(21), \\
n=1221 \\
486(35), \\
n=1346\end{array}$ & $\begin{array}{l}527(51), \\
n=112 \\
529(46), \\
n=91\end{array}$ \\
\hline Exp. 2 & $\begin{array}{l}\text { Short ITI } \\
(500 \mathrm{~ms}) \\
\text { Long ITI } \\
(3000 \mathrm{~ms})\end{array}$ & $\begin{array}{l}457(36), \\
n=1096 \\
499(41), \\
n=1317\end{array}$ & $\begin{array}{l}465(57), \\
n=142 \\
492(58), \\
n=97\end{array}$ & $\begin{array}{l}463(24), \\
n=1121 \\
496(33), \\
n=1351\end{array}$ & $\begin{array}{l}513(50) \\
n=126 \\
501(51) \\
n=91\end{array}$ \\
\hline \multicolumn{6}{|c|}{ PIA: Errors (\%) } \\
\hline Exp. 1 & $\begin{array}{l}\text { Short ITI } \\
(500 \mathrm{~ms}) \\
\text { Long ITI } \\
(1700 \mathrm{~ms})\end{array}$ & $\begin{array}{l}16.7(6.3), \\
n=1438 \\
11.5(8.4), \\
n=1515\end{array}$ & $\begin{array}{l}16.8(10.6) \\
n=227 \\
10.0(14.2) \\
n=144\end{array}$ & $\begin{array}{l}11.7(10.4) \\
n=1444 \\
7.6(9.1) \\
n=1499\end{array}$ & $\begin{array}{l}16.9(14.4), \\
n=220 \\
20.2(26.1), \\
n=151\end{array}$ \\
\hline Exp. 2 & $\begin{array}{l}\text { Short ITI } \\
(500 \mathrm{~ms}) \\
\text { Long ITI } \\
(3000 \mathrm{~ms})\end{array}$ & $\begin{array}{l}19.4(6.4), \\
n=1383 \\
11.5(8.2), \\
n=1494\end{array}$ & $\begin{array}{l}18.0(13.0) \\
n=241 \\
5.9(8.9) \\
n=139\end{array}$ & $\begin{array}{l}14.0(8.0), \\
n=1373 \\
8.1(8.0), \\
n=1483\end{array}$ & $\begin{array}{l}21.7(20.0), \\
n=240 \\
7.3(10.1), \\
n=146\end{array}$ \\
\hline
\end{tabular}

Note. Mean reaction times (RTs) and error rates for correct and incorrect responses are displayed separately for the response correctness $n-1$ (post-correct vs. post-error), ITI (intertrial interval; short vs. long) conditions, and response target (repeat vs. switch) in Experiments 1 and 2. PES indicates post-error slowing and PIA post-error increased accuracy. Mean RTs (in ms) and mean error rates (in \%) are displayed with corresponding within subject standard deviations (corrected according to Morey, 2008) in brackets followed by the number of trials per cell ( $n$ ). 
PES. Table 2 shows the results and model statistics of the RT model. For participants, the estimated random intercept variance was $770 \mathrm{~ms}$. The variance for the by-participants random effects for response-correctness $n-1$ was $27 \mathrm{~ms}$, for ITI $120 \mathrm{~ms}$, for target type $100 \mathrm{~ms}$, and $137 \mathrm{~ms}$ for the interaction between response-correctness $n$-1 and target type, 20ms for the interaction between response-correctness $s_{n-1}$ and ITI, and $4 \mathrm{~ms}$ for the interaction between ITI and target type. Comparing post-error and post-correct trials, the LMM on RTs yielded a strong and significant effect of response-correctness $\mathrm{s}_{n-1}$, with higher RTs in correct trials following an error (post-error) than post-correct trials $(2 \times b=40 \mathrm{~ms}$; see Figure $3 \mathrm{~A}+3 \mathrm{~B})$. Furthermore, the LMM revealed a significant effect of ITI, indicating faster RTs for short ITIs of 500ms than for long ITIs of $1700 \mathrm{~ms}(2 \times b=16 \mathrm{~ms})$.

Participants responded significantly faster for response repeat than for response switch targets $(2 \times b=-21 \mathrm{~ms})$ and their RTs became faster with increasing trial number $(b=-11 \mathrm{~ms})$. Importantly, the interaction of ITI and response-correctness $\mathrm{n}_{\mathrm{n}-1}$, did not reach statistical significance. Yet, as hypothesized, the effect of response-correctness $\mathrm{n}_{\mathrm{n}-1}$ was significantly stronger in response switch as compared to response repeat targets. Post-hoc comparisons revealed that RTs were significantly higher in post-error as compared to post-correct trials for response switch targets $($ difference $=58 \mathrm{~ms}, p<.001)$ as well as response repeat targets $($ difference $=23 \mathrm{~ms}$, $p=.022)$. We found no evidence of an influence of participants' error awareness on responsecorrectness $_{\mathrm{n}-1}$ and no three-way interaction between response-correctness $s_{\mathrm{n}-1}$, ITI, and target type. 
Table 2

Results of the Linear Mixed Model for the Reaction Times (RTS) in Experiment 1

\begin{tabular}{|c|c|c|c|c|c|c|}
\hline \multicolumn{7}{|c|}{ RT-Model } \\
\hline Predictors & Estimate & $C I$ & SE & $d f$ & $t$-value & $p$ \\
\hline (Intercept) & 491.10 & $479.19-503.01$ & 6.08 & 25.52 & 80.82 & $<.001$ \\
\hline $\begin{array}{l}\text { Response- } \\
\text { Correctness }_{\mathrm{n}-1} \text { (error) }\end{array}$ & 20.08 & $15.29-24.87$ & 2.44 & 22.93 & 8.22 & $<.001$ \\
\hline ITI (short) & -6.98 & $-13.06--0.89$ & 3.10 & 39.31 & -2.25 & .025 \\
\hline Target Type (repeat) & -10.53 & $-16.40--4.65$ & 3.00 & 28.97 & -2.55 & $<.001$ \\
\hline Error-Awareness & -20.52 & $-32.66--8.37$ & 6.20 & 26.48 & -3.31 & .001 \\
\hline $\mathrm{N}_{\text {Block }}$ & -11.20 & $-15.51--6.89$ & 2.20 & 52.04 & -5.10 & $<.001$ \\
\hline $\begin{array}{l}\text { Response- } \\
\text { Correctness }_{n-1} \text { x ITI }\end{array}$ & 4.35 & $-0.26-8.96$ & 2.35 & 35.60 & 1.85 & .064 \\
\hline $\begin{array}{l}\text { Response- } \\
\text { Correctness } n-1 \text { x Target } \\
\text { Type }\end{array}$ & -8.75 & $-15.11--2.38$ & 3.25 & 31.29 & -2.69 & .007 \\
\hline ITI x Target Type & -0.16 & $-4.44-4.11$ & 2.18 & 195.06 & -0.07 & .941 \\
\hline $\begin{array}{l}\text { Response- } \\
\text { Correctness }_{\mathrm{n}-1} \mathrm{X} \\
\text { Error-Awareness }\end{array}$ & 4.18 & $-0.91-9.27$ & 2.60 & 25.62 & 1.61 & .108 \\
\hline $\begin{array}{l}\text { Response- } \\
\text { Correctness } \text { n-1 } \text { x ITI x } \\
\text { Target Type }\end{array}$ & -1.35 & $-5.55-2.85$ & 2.14 & 3832.00 & -0.63 & .529 \\
\hline \multicolumn{7}{|l|}{ Model } \\
\hline Marginal $\mathrm{R}^{2}$ / Condition & al $R^{2}$ & $0.107 / 0.195$ & & & & \\
\hline Observations & & 5528 & & & & \\
\hline
\end{tabular}

Note. $\mathrm{CI}=95 \%$ confidence intervals. 


\section{Figure 3}

Main Results of Experiment 1

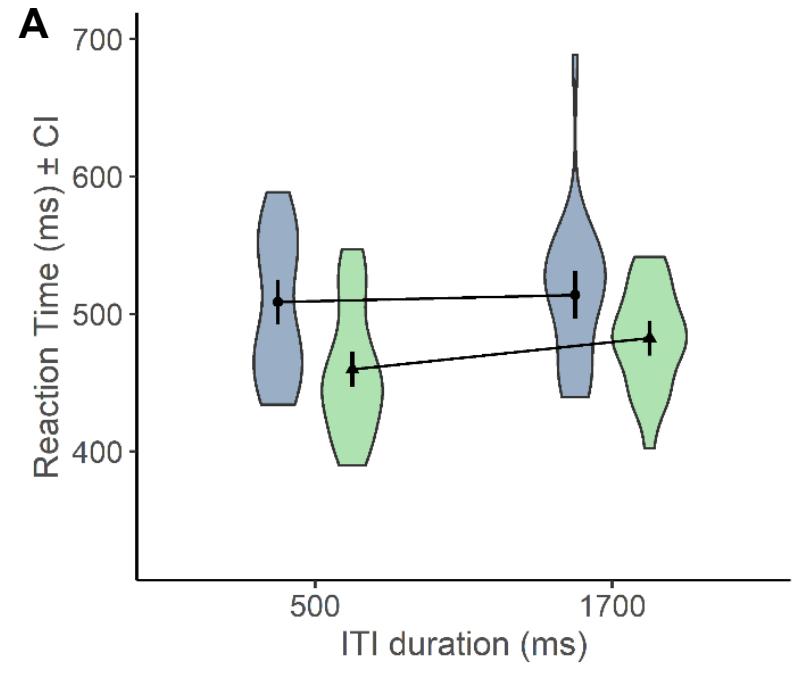

Current Trial: $\bullet$ post-error $\Delta$ post-correct

C

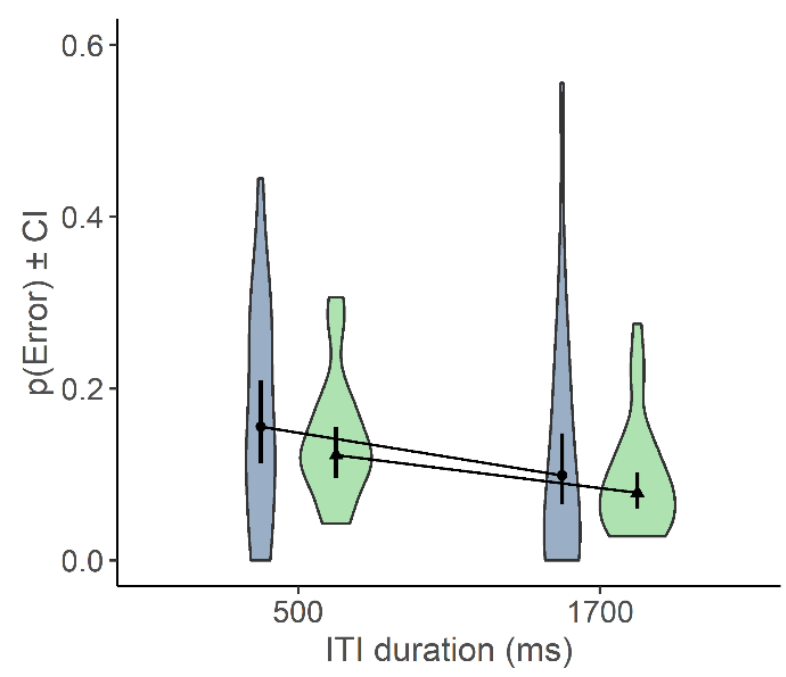

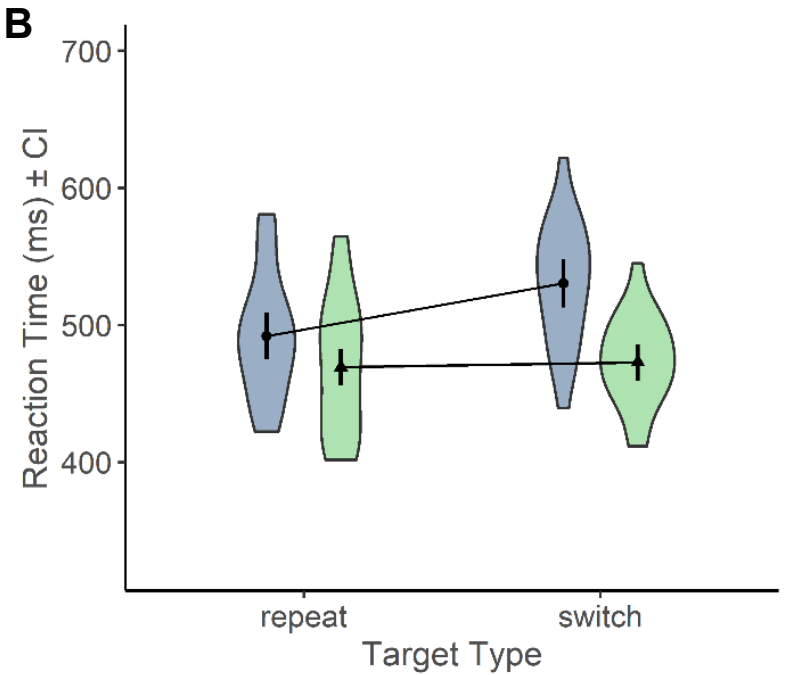

Current Trial: $-\infty$ post-error $\Delta$ post-correct

D

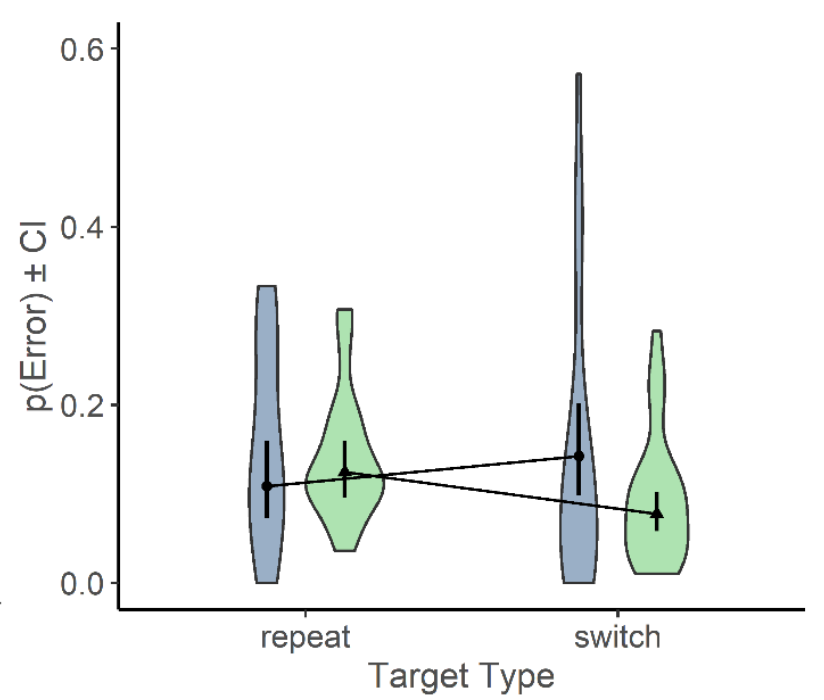

Note. Mean reaction times in correct trials are plotted as a function of response-correctness n-1 and A) intertrial, ITI, duration or B) target type. Mean error rates are plotted as a function of response-correctness $\mathrm{n}-1$ and C) ITI duration or D) target type. Error bars denote the modelbased $95 \%$ confidence intervals $(\mathrm{CI})$. 
PIA. Results and model statistics of the error-model are displayed in Table 3. For participants, the estimated variance for the by-participants random intercept was 0.31 , for the random slopes response-correctness $\mathrm{s}_{n-1} 0.07$, target type 0.05 , ITI 0.004 , and interaction between responsecorrectness $_{n-1}$ and target type 0.02 .

Table 3

Results for the Generalized Linear Mixed Model for Errors in Experiment 1

\begin{tabular}{|c|c|c|c|c|c|c|}
\hline \multicolumn{7}{|c|}{ Error-Model } \\
\hline Predictors & $\begin{array}{c}\text { Odds } \\
\text { Ratios }\end{array}$ & $C I$ & Estimate & $\mathrm{SE}$ & $z$-value & $p$ \\
\hline Intercept & 0.12 & $0.10-0.16$ & -2.08 & 0.02 & -15.59 & $<.001$ \\
\hline Response-Correctness $\mathrm{n}_{\mathrm{n}-1}$ (error) & 1.14 & $0.96-1.35$ & 0.13 & 0.10 & 1.54 & .124 \\
\hline ITI (short) & 1.29 & $1.14-1.45$ & 0.25 & 0.08 & 4.15 & $<.001$ \\
\hline Target Type (repeat) & 1.06 & $0.91-1.23$ & 0.06 & 0.08 & 0.72 & .473 \\
\hline Error-Awareness & 0.90 & $0.69-1.16$ & -0.11 & 0.12 & -0.82 & .413 \\
\hline NBlock & 0.99 & $0.91-1.08$ & -0.01 & 0.04 & -0.23 & .822 \\
\hline Response-Correctness $s_{n-1}$ X ITI & 1.01 & $0.90-1.13$ & 0.01 & 0.06 & 0.11 & .915 \\
\hline $\begin{array}{l}\text { Response-Correctness } \mathrm{n}_{\mathrm{n}-1} \mathrm{x} \\
\text { Target Type }\end{array}$ & 0.81 & $0.71-0.93$ & -0.21 & 0.06 & -3.06 & .002 \\
\hline ITI x Target Type & 1.08 & $0.96-1.21$ & 0.08 & 0.06 & 1.28 & .201 \\
\hline $\begin{array}{l}\text { Response-Correctness }_{\mathrm{n}-1} \mathrm{X} \\
\text { Error-Awareness }\end{array}$ & 0.81 & $0.69-0.97$ & -0.21 & 0.07 & -2.36 & .018 \\
\hline $\begin{array}{l}\text { Response-Correctness } \mathrm{n}_{\mathrm{n}-1} \mathrm{x} \text { ITI } \\
\mathrm{x} \text { Target Type }\end{array}$ & 1.08 & $0.96-1.21$ & 0.07 & 0.06 & 1.29 & .197 \\
\hline Model & & & & & & \\
\hline Marginal $\mathrm{R}^{2}$ / Conditional $\mathrm{R}^{2}$ & \multicolumn{2}{|c|}{$0.041 / 0.124$} & & & & \\
\hline Observations & \multicolumn{2}{|c|}{6638} & & & & \\
\hline
\end{tabular}

Note. CI indicates $95 \%$ confidence intervals. 
Our model neither supported the assumption of a regular or an inversed PIA effect (increased likelihood to commit an error in trials following an error), as the GLMM did not yield a significant effect of response-correctness $\mathrm{n}_{\mathrm{n}}$ (PIA, see Figure 3C). There was no significant interaction between response-correctness $\mathrm{n}_{\mathrm{n}-1}$ and ITI $(O R=1.01)$. However, as predicted, we found a significant interaction between response-correctness $n-1$ and target type. A post-hoc analysis of this interaction indicated an inversed PIA effect for response switch targets $(p=.024)$, but not for response repeat targets $(p=0.856)$ as illustrated in Figure 3D.

There was a significant effect of ITI duration suggesting that the likelihood to commit an error was higher in trials with short rather than with long ITIs $(O R=1.29)$. Moreover, while error-awareness did not generally impact the likelihood to commit an error (no significant main effect), we found a significant interaction between error awareness and response-correctness $n-1$ $(O R=0.81):$ When error-awareness was low, participants appeared to have an increased likelihood to commit an error following error trials.

\section{Discussion}

In Experiment 1, participants' to-be-performed response in a given trial $\mathrm{n}_{\mathrm{n}}$ either repeated or switched as compared to the correct response of the preceding trial $\mathrm{n}_{\mathrm{n}-1}$. At the same time, we manipulated the preparation time after an error (short ITI $=500 \mathrm{~ms}$, long ITI $=1700 \mathrm{~ms} /$ short RSI $=1300 \mathrm{~ms}$, long RSI $=2500 \mathrm{~ms}$ ). The aim of the Experiment 1 was to test whether posterror cognitive control resulted in a disruption of the current task-set related representation (memory obstruction hypothesis). To test this assumption, we assessed whether participants showed an inversed PIA effect that differed between target types. Furthermore, we assessed whether PIA and PES effects changed with ITI duration. 
To check whether error-related processes as well as influences of RAA in our design were comparable to prior studies on post-error processing not using response repeat/switch targets, we analyzed whether participants demonstrated RAA and a typical PES for short ITIs. In line with prior research, we found both a distinct PES effect (Notebaert et al., 2009; Rabbitt, 1966) and high RAAs for incorrect and correct responses comparable to prior studies (e.g., Boldt \& Yeung, 2015; Grützmann et al., 2014; Klein et al., 2013; Steinhauser \& Yeung, 2010). At the same time, in line with our expectations and trends found in prior research (Grützmann et al., 2014), RAA was higher for correct as compared to incorrect responses. Thus, post-error adaptation effects for short ITIs (here, the orienting-related PES, Wessel, 2018) and RAA were comparable to prior studies on post-error processing. That is, the absence or inversion of the PIA effect as well as differences between target types and the absence of an influence of ITI on PES/PIA we predicted cannot be attributed to confounding basic paradigmatic differences between our experiment and prior studies on post-error processing.

PES. RTs were higher in post-error trials as compared to post-correct trials for both short and long ITIs. That is, ITI duration did not significantly impact on the PES effect. Note, that this result distinctively differs from prior studies which typically found that PES decreases with increasing ITI (e.g., Buzzell et al., 2017; Jentzsch \& Dudschig, 2009; Ullsperger \& Danielmeier, 2016; van der Borght et al., 2016). Thus, as hypothesized and contrary to prior research, our results do not provide any evidence that PES decreased with increasing ITI. As detailed in the introduction, this suggests that controlled post-error processing (e.g., the reinstatement of the task-set) may take longer or never be fully completed in the current experiment in which response repeat/switch targets were used. Thus, this finding provides first support for the memory obstruction hypothesis. Alternatively, and also in line with Wessel's theory (2018), the 
prolonged RTs following an error measured in the long ITI could also reflect a form of strategic PES instead of or in addition to orienting-related PES (see Wessel, 2018). For instance, when the preceding error was caused by insufficient preparation for the response repeat/switch target, it may be beneficial for participants to slow down after an error to increase preparation time.

Furthermore, as predicted, PES was stronger for response switch as compared to response repeat targets, suggesting that costs associated with the disruption of the current task-set related representations were stronger in response switch than in response repeat trials. We explain this as follows: In our design, response switch targets generally necessitate a multi-step process to determine the correct response $\left(1^{\text {st }}\right.$ : retrieve reference response, $2^{\text {nd. }}:$ invert reference response), whereas a single step is sufficient on response repeat trials (retrieve reference response) ${ }^{4}$. It was most likely for participants to initially adapt the reference response after an error (given the long RSI and unpredictability of upcoming targets). These processes should therefore have been required both post-correct response as well as post-error. Our finding is thus in line with the idea

${ }^{4}$ Note, that, in post-error trials, a response switch target required the same response as given on the previous trial, whereas a response repetition target required the opposite response. We cannot rule out that participants built long-term representations that facilitated the inversion of the reference response in post-error trials (e.g., "if error and switch, then same response"). To do so, however, one would need to assume that participants integrated multiple long-term associations (vowel/consonant-repeat/switch and error - repeat/switch reversal) with information on the prior response. We consider a more sequential processing (as described above) as equally parsimonious and more likely given participants' ample preparation time during the ITI/RSI. 
that the increased cognitive resource demands for response switch targets add to difficulties reconstructing the obstructed task-set related memory in post-error trials.

$\boldsymbol{P I A}$. Although we did not find a significant inversed PIA effect, there also was no evidence of a PIA effect which is in line with a weak interpretation of the memory obstruction hypothesis. Supporting this conclusion, as predicted, we found no evidence for an interaction between PIA and ITI. This pattern of results predicted by the memory obstruction hypothesis is in stark contrast with prior studies on post-error adaptations that typically found a PIA effect for long but not short ITIs (e.g., Buzzell et al., 2017; Jentzsch \& Dudschig, 2009). Finally, as predicted, target type and PIA interacted and in response switch trials we found evidence for an inversed PIA effect. Thus, the pattern of results in our experiment as compared to prior studies is consistently confirming the memory obstruction hypothesis (though not in its strongest version, see following discussion).

Importantly, however, accuracy in post-error trials was nowhere near chance performance (error rates in short ITI: $M=16.9 \%$; error rates in long ITI: $M=11.8 \%$ ). Error rates showed that participants could still reinstate the task-set related information in the present experiment and respond correctly on the majority of trials. Yet, we found no evidence that participants' accuracy increased following an error and it even decreased following an error when a response switch was required. This strongly suggests a disruption of task-set related representations in WM following an error (see the General Discussion for a more detailed discussion of contributions from LTM and WM). Yet, it suggests that the task-set related representations in WM (task-set translations and/or past responses) were either not disrupted completely and/or could be reinstated nonetheless. Thus, the pattern of results is in favor of the weak interpretation of the memory obstruction hypothesis: Although performance was not at chance level, participants 
were unable to improve in trials following an error (contrary to prior research: e.g., Maier et al., 2011). Crucially, this was true for both short and long ITIs and thus confirms the assumption of the memory obstruction hypothesis that, even at long ITIs (note that both ITIs/RSIs were even substantially longer than in prior studies: e.g., e.g., Buzzell et al., 2017; Jentzsch \& Dudschig, 2009), participants fail to improve their accuracy following errors when response repeat/switch targets hinder task-set reconstruction. In addition, the observation that performance in post-error trials was not at chance level even at short ITIs questions the idea that task-set related representations were completely inhibited or removed from memory at that time.

Taken together, our results are thus in line with a weak version of the memory obstruction hypothesis: It seems that after an error, the current task-set related representations in WM (or at least any proactively prepared tasks-set translations or memory for the previous response) were disrupted but not completely removed from memory. Experiment 1 provided evidence for the assumption that post-error inhibitory control obstructs (working) memory representations of the current task-set related representations (as proposed by Wessel, 2018) which counteracts any PIA effects when response repeat/switch targets are used and renders preparation time during the ITI inconsequential. Yet, it seems that the task-set could nevertheless be restored in most cases as performance was clearly better than chance speaking against a strong interpretation of the memory obstruction hypothesis.

This begs the question whether participants would simply need more preparation time (i.e., longer ITIs) to fully restore the task-set and show, for instance, PIA effects even when response repeat/switch targets are used. That is, whether increased cognitive demands in our paradigm might just lead to a need for increased preparation time. Possibly, post-error adaptions based on controlled post-error processing could still successfully operate when given more time. 
In Experiment 2, we thus aimed to first replicate our findings and further test the memory obstruction hypothesis of post-error cognitive control by giving participants even more time to recover after an error.

\section{Experiment 2}

The aim of Experiment 2 was to replicate the findings of Experiment 1 with the notable following difference: The long ITI duration was increased to $3000 \mathrm{~ms}(\mathrm{RSI}=3800 \mathrm{~ms})$ to give participants more time to re-orient their attention to the current task-set related information and update the task-set translations according to the previous trial. Based on the memory obstruction hypothesis, we tested whether participants would - at best - show post-error performance improvements when given more time, whereas post-error decrements in accuracy (or at least no improvements for response repeat targets) were expected for the short ITI.

\section{Method (Participants \& Procedure)}

The same exclusion criteria as in Experiment 1 were applied. For the second experiment, from an initial sample size of $N=25$ German participants taking part for course credit or monetary compensation, one participant had to be excluded due to an error rate above $50 \%(n=$ 1). This resulted in a sample size of $n=24$ participants for analyses $\left(M_{\text {age }}=26.64, S D=6.55\right.$, range $20-51$ years; $72 \%$ female, $28 \%$ male). Again, all participants were naive to the purpose of the experiment, had normal or corrected-to-normal vision, and gave written informed consent prior to participating. The experimental set-up and the procedure were identical to Experiment 1 with one exception: The ITI was either 500 ms (RSI: 1300 ms) or 3000 ms (RSI: 3800 ms).

\section{Results}

Mean RTs and error rates can be taken from Table 1 . The statistical analyses, the corresponding software, and preprocessing procedures were the same as in Experiment 1. Trials 
with response omissions $(n=296,2.5 \%)$, and premature responses $(n=44,0.4 \%)$ including trials preceding and following these responses (response omission: $n=523,4.4 \%$, premature response: $n=78,0.6 \%$ ) were excluded from all analyses. The overall error rate in test-blocks was $13.9 \%(S D=7.7 \%)$ and thus comparable to Experiment 1. As in Experiment 1, error rates in RAA-blocks were higher $(19.3 \%, S D=12.4 \%)$ than in test-blocks, $V=277, p<.001$. There were no participants with exceptionally high (> $3 \mathrm{SDs}$ ) error rates.

\section{Response Accuracy Awareness (RAA)}

The evaluation of participants' RAA showed that erroneous responses were again consistently rated as incorrect $(M=75.4 \%, S D=24.0 \% ; M D N=83.3 \% ; V=277, p<.001)$ and correct responses as correct $(M=92.0 \%, S D=8.1 \% ; M D N=94.2 \% ; V=300, p<.001)$ above chance. Again, RAA was higher for correct than for incorrect responses with a Median difference of $12.5 \%(V=300, p<.001)$. Wilcoxon signed rank tests were used for all RAA analyses as deviations from normality occurred (all $p \leq .013$ ).

\section{PES and PIA}

Data analysis was equivalent to Experiment 1. Trial sequences yielding sequences of error repetitions including the trials preceding and directly following such error sequences $(n=$ $663,10.0 \%$ ) were excluded for the analysis of PES. For the analysis of PIA the first two repeating errors were kept and only the following trials of the error sequence and the trial following the end of the sequence were deleted $(n=147,2.2 \%)$. Additionally, for PES, we did not consider trials which were in-between errors and thus rated as both a post- and pre-error trial $(n=55,0.0 \%)$. Furthermore, for PES, trials with RTs deviating more than three standard deviations (SDs) from the mean RT within each condition (PES: $n=30,0.6 \%$ ) were excluded.

PES. The RT model was identical to Experiment 1. Results and model statistics for the RT model can be taken from Table 4. For participants, the estimated random intercept variance 
was $770 \mathrm{~ms}$. The by-participant random effects variance of response-correctness $\mathrm{s}_{n-1}$ was $27 \mathrm{~ms}$, of ITI $120 \mathrm{~ms}$, of target type $100 \mathrm{~ms}$, and $20 \mathrm{~ms}$ for the interaction between response-correctness $n-1$ and ITI, $4 \mathrm{~ms}$ for the interaction between target type and ITI as well as $137 \mathrm{~ms}$ for the interaction between response-correctness $s_{n-1}$ and target type.

\section{Table 4}

Results for the Linear Mixed Model of the Reaction Times (RTS) in Experiment 2

\begin{tabular}{|c|c|c|c|c|c|c|}
\hline \multicolumn{7}{|c|}{ RT-Model } \\
\hline Predictors & Estimate & $C I$ & Std. Error & $d f$ & $t$-value & $p$ \\
\hline Intercept & 486.28 & $465.11-507.45$ & 10.80 & 22.66 & 45.02 & $<.001$ \\
\hline $\begin{array}{l}\text { Response-Correctness } \mathrm{n}_{\mathrm{n}-1} \\
\text { (error) }\end{array}$ & 7.06 & $0.60-13.52$ & 3.30 & 24.14 & 2.14 & .032 \\
\hline ITI (short) & -9.62 & $-18.66--0.59$ & 4.61 & 27.49 & -2.09 & .037 \\
\hline Target Type (repeat) & -8.02 & $-14.29--1.75$ & 3.20 & 45.13 & -2.51 & .012 \\
\hline Error-Awareness & 12.38 & $-8.74-33.49$ & 10.77 & 22.73 & 1.15 & .251 \\
\hline NBlock & -3.86 & $-9.53-1.82$ & 2.90 & 143.12 & -1.33 & .183 \\
\hline $\begin{array}{l}\text { Response-Correctness } \mathrm{n}_{\mathrm{n}-1} \\
\text { x ITI }\end{array}$ & 8.88 & $3.26-14.50$ & 2.87 & 27.18 & 3.10 & .002 \\
\hline $\begin{array}{l}\text { Response-Correctness } \mathrm{n}_{\mathrm{n}-1} \\
\mathrm{x} \text { Target Type }\end{array}$ & -7.16 & $-11.73--2.59$ & 2.33 & 40.33 & -3.07 & .002 \\
\hline ITI x Target Type & -3.07 & $-7.40-1.26$ & 2.21 & 3757.27 & -1.39 & .164 \\
\hline $\begin{array}{l}\text { Response-Correctness } \mathrm{n}-1 \\
\mathrm{x} \text { Error-Awareness }\end{array}$ & 7.79 & $1.28-14.29$ & 3.32 & 25.24 & 2.35 & .019 \\
\hline $\begin{array}{l}\text { Response-Correctness } n-1 \\
\text { x ITI x Target Type }\end{array}$ & -0.71 & $-4.98-3.57$ & 2.18 & 3912.99 & -0.32 & .746 \\
\hline \multicolumn{7}{|l|}{ Model } \\
\hline \multicolumn{2}{|c|}{ Marginal $\mathrm{R}^{2}$ / Conditional $\mathrm{R}^{2}$} & \multicolumn{5}{|l|}{$0.042 / 0.304$} \\
\hline Observations & & \multicolumn{5}{|l|}{5341} \\
\hline
\end{tabular}

Note. CI indicates $95 \%$ confidence intervals. 
Comparing post-error and post-correct trials, the LMM on RTs yielded again a significant effect of response-correctness $n$-1 , with higher RTs in correct trials following an error (post-error) than post-correct trials $(2 \times b=14 \mathrm{~ms}$; see Figure 4A $+4 \mathrm{~B})$. Again, RTs were faster for short ITIs of $500 \mathrm{~ms}$ than for long ITIs of $3000 \mathrm{~ms}(2 \times b=-20 \mathrm{~ms})$ and faster for response repeat targets than response switch targets $(2 \times b=-16 \mathrm{~ms})$. Other than in Experiment 1, the effect of responsecorrectness $_{n-1}$ significantly interacted with the ITI duration. In trials with a short ITI a significant PES effect was apparent (post-hoc comparisons for short ITIs between post-correct and posterror trials: difference $=32 \mathrm{~ms} ; p<.001$ ), whereas there was no significant PES effect in trials with a long ITI of 3000ms (post-hoc comparisons for long ITIs between post-correct and posterror trials: difference $=-4 \mathrm{~ms} ; p=.980$ ). Yet, as also evident in the post-hoc comparisons and Figure 4A, this interaction effect was not driven by a decrease in RTs in post-error trials in the $3000 \mathrm{~ms}$ as compared to the $500 \mathrm{~ms}$ ITI duration (difference $=-2 \mathrm{~ms} ; p=.999$ ), but by an increase in RTs for post-correct trials for long ITI durations (difference $=-37 \mathrm{~ms} ; p<.001$ ). Thus, we found a general slowing effect for long as compared to short ITIs for post-correct trials, but this slowing effect was reduced in post-error trials.

As predicted, we again found an interaction between response-correctness $\mathrm{s}_{\mathrm{n}-1}$ and target type as also illustrated in Figure 4B. Post-hoc comparisons revealed that the effect of responsecorrectness $_{\mathrm{n}-1}$ was stronger for response switch than for response repeat targets as there was no significant difference between post-error and post-correct trials for response repeat targets (difference: $0 \mathrm{~ms}, p=1.00$ ). For response switch targets, however, participants responded $29 \mathrm{~ms}$ slower in post-error as compared to post-correct trials $(p=.003)$. 
Figure 4

Main Results of Experiment 2

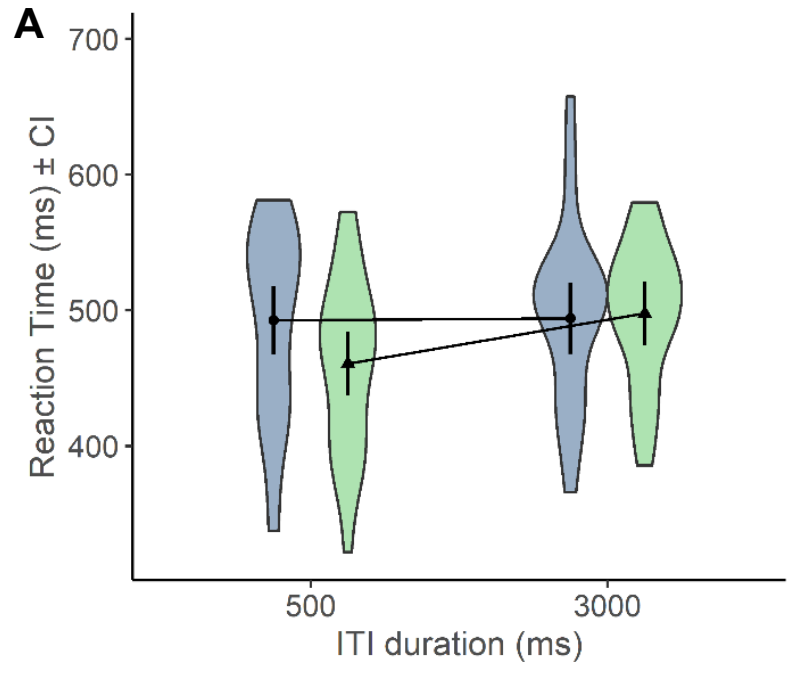

Current Trial $\bullet$ post-error $\Delta$ post-correct

C

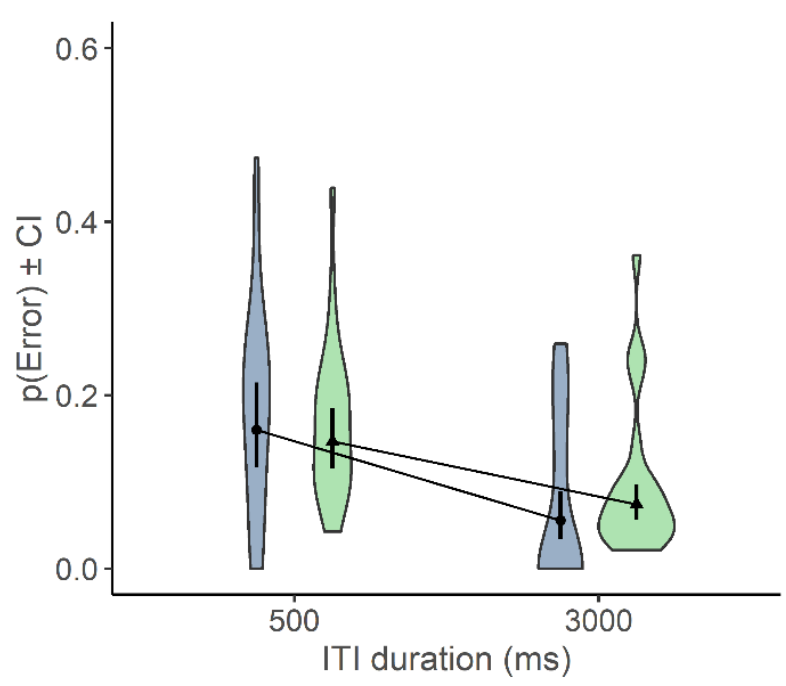

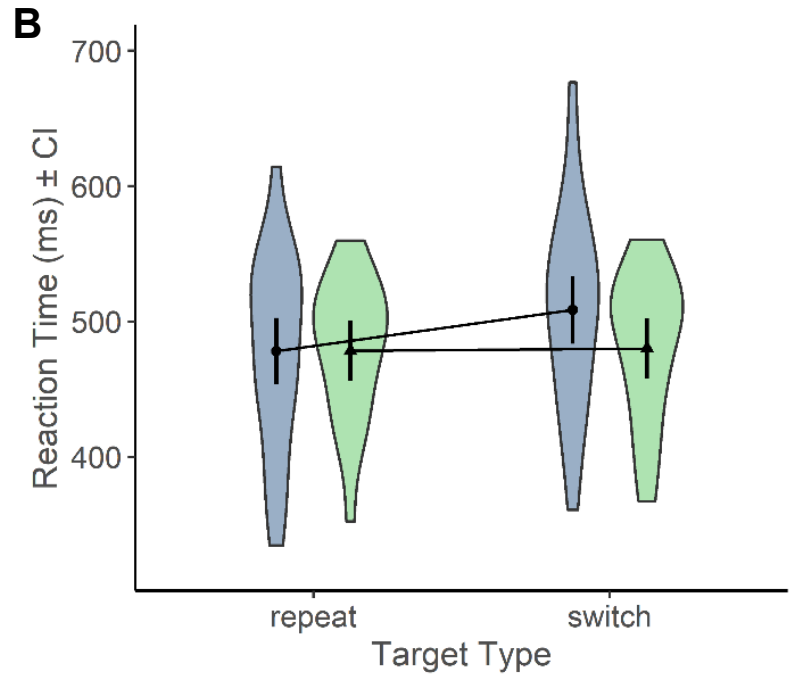

Current Trial $\bullet$ post-error $\Delta$ post-correct

D

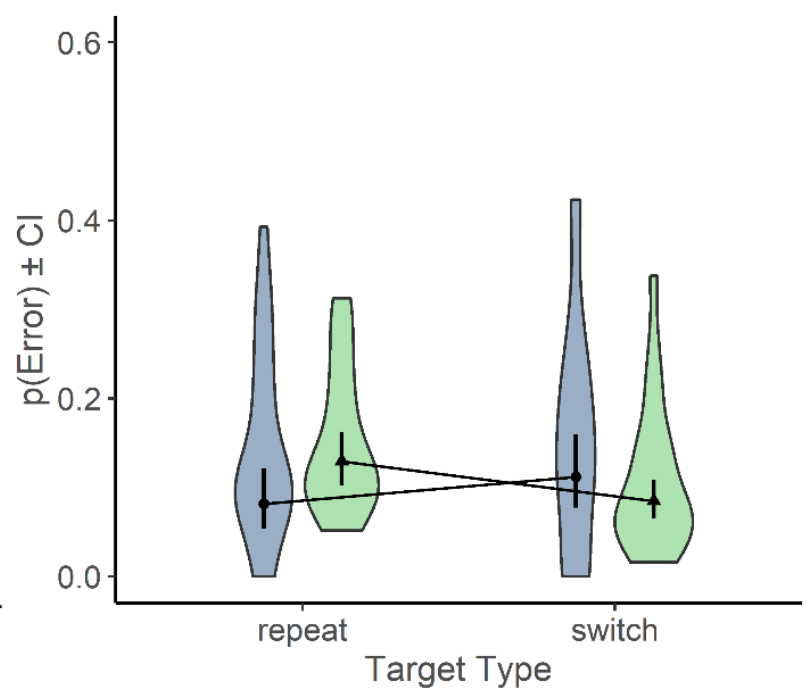

Note. Mean reaction times in correct trials are plotted as a function of response-correctness n-1 and A) intertrial, ITI, duration or B) target type. Mean error rates are plotted as a function of response-correctness n-1 and C) ITI duration or D) target type. Error bars denote the modelbased $95 \%$ confidence intervals (CI). 
Moreover, and in contrast to Experiment 1, we found a significant interaction between response-correctness $\mathrm{n}_{\mathrm{n}-1}$ and error awareness $(b=8 \mathrm{~ms})$ : Participants with high error awareness demonstrated increased PES effects as compared to participants with a low error awareness.

PIA. The error model was identical to Experiment 1 except that it did not include a byparticipant random slope for the interaction between target type and response-correctness $n-1$. Results and model statistics of the error model can be taken form Table 5. The effects of the error-model are illustrated in Figure 4C $+4 \mathrm{D}$. For participants, the estimated random intercept variance was 0.31 . The estimated random effects variances of the by-participants random slopes were 0.004 for ITI, for response-correctness $\mathrm{n}_{-1} 0.07$, and 0.05 for the target type.

Again, our model did not support the assumption of a regular or an inversed PIA effect (increased likelihood to commit an error in trials following an error), as the GLMM did not yield a significant effect of response-correctness $n$-1. In addition, we found no evidence for a significant interaction between response-correctness $\mathrm{n}_{\mathrm{n}-1}$ and ITI. Yet again, response-correctness $\mathrm{n}_{\mathrm{n}-1}$ significantly interacted with target type $(O R=0.81)$. This time, the post-hoc analyses indicated a different pattern: In contrast to Experiment 1, there was no inversed PIA effect for response switch targets $(p=.309)$, but a typical PIA effect for response repeat targets $(p=.048)$. In addition, in post-correct $(p=.001)$ but not in post-error trials $(p=.488)$, participants committed fewer errors for response switch as compared to response repeat targets as illustrated in Figure 4D. There was a significant effect of ITI duration suggesting that the likelihood to commit an error was higher in trials with short rather than with long ITIs $(O R=1.63)$. In contrast to Experiment 1, the interaction between the response-correctness $\mathrm{n}_{\mathrm{n}-1}$ and error awareness failed to reach significance in the error model. 
Table 5

Results for the Generalized Linear Mixed Model for Errors in Experiment 2.

\begin{tabular}{|c|c|c|c|c|c|c|}
\hline \multicolumn{7}{|c|}{ Error Model } \\
\hline \multirow{2}{*}{$\frac{\text { Predictors }}{\text { Intercept }}$} & \multirow{2}{*}{$\begin{array}{l}\begin{array}{l}\text { Odds } \\
\text { Ratios }\end{array} \\
0.11\end{array}$} & \multirow{2}{*}{$\begin{array}{l}C I \\
0.08-0.15\end{array}$} & \multicolumn{2}{|c|}{ Estimate SE } & \multirow{2}{*}{$\begin{array}{r}z \text {-value } \\
-16.23\end{array}$} & \multirow{2}{*}{$\begin{array}{l}p \\
<.001\end{array}$} \\
\hline & & & -2.20 & 0.02 & & \\
\hline Response-Correctness $_{\mathrm{n}-1}$ (error) & 0.95 & $0.82-1.10$ & -0.05 & 0.07 & -0.71 & .479 \\
\hline ITI (short) & 1.63 & $1.35-1.95$ & 0.49 & 0.15 & 5.19 & $<.001$ \\
\hline Target Type (repeat) & 1.03 & $0.89-1.20$ & 0.03 & 0.08 & 0.39 & .694 \\
\hline Error-Awareness & 0.75 & $0.58-0.97$ & -0.29 & 0.10 & -1.21 & .027 \\
\hline NBlock & 0.87 & $0.76-1.00$ & -0.14 & 0.06 & -2.03 & .043 \\
\hline Response-Correctness $\mathrm{n}_{\mathrm{n}-1}$ x ITI & 1.11 & $0.98-1.26$ & 0.10 & 0.07 & 1.62 & .106 \\
\hline $\begin{array}{l}\text { Response-Correctness }_{\mathrm{n}-1} \mathrm{X} \\
\text { Target Type }\end{array}$ & 0.81 & $0.72-0.92$ & -0.21 & 0.05 & -3.38 & .001 \\
\hline ITI x Target Type & 1.00 & $0.89-1.13$ & -0.00 & 0.06 & -0.03 & .980 \\
\hline $\begin{array}{l}\text { Response-Correctness } \mathrm{n}_{\mathrm{n}-1} \mathrm{X} \\
\text { Error-Awareness }\end{array}$ & 0.89 & $0.78-1.01$ & -0.12 & 0.06 & -1.82 & .069 \\
\hline $\begin{array}{l}\text { Response-Correctness } \mathrm{n}_{\mathrm{n}-1} \text { x ITI } \\
\mathrm{x} \text { Target Type }\end{array}$ & 1.00 & $0.89-1.12$ & -0.00 & 0.06 & -0.04 & .972 \\
\hline \multicolumn{7}{|l|}{ Model } \\
\hline Marginal $\mathrm{R}^{2}$ / Conditional $\mathrm{R}^{2}$ & \multicolumn{2}{|c|}{$.070 / 0.148$} & & & & \\
\hline Observations & 6499 & & & & & \\
\hline
\end{tabular}

Note. $\mathrm{CI}=$ confidence intervals. 


\section{Pooled Bayesian Analysis: Testing the influence of ITI duration on Post-Error Accuracy}

To further substantiate the evidence against an influence of ITI on the PIA effect in our experiments, we additionally analyzed the data of Experiments 1 and 2 (preprocessing was the same as before) post-hoc in a pooled analysis using Bayesian generalized linear mixed models (BGLMMs) implemented in the R package brms (Bürkner, 2017, 2018). We decided to use a Bayesian approach for this analysis, because we explicitly wanted to assess the evidence for the null against the alternative hypothesis. PIA was again assessed by predicting the correctness of responses (incorrect response $=1$, correct response $=0$ ) assuming a Bernoulli data distribution predicted by a linear model through a logistic link function and using the fixed factors ITI duration $_{(1}=$ short/500 ms vs. $-1=$ long/1700/3000 ms $)$, response-correctness $n-1$ ( 1 = posterror, -1 = post-correct $)$, and experiment $(1=$ data from Experiment $1,-1=$ data from Experiment 2) as well as all corresponding interactions between these three factors. In addition, our model included the fixed factor target type $(1=$ repeat vs. $-1=$ switch $)$ and an interaction between the factors target type and response-correctnes $s_{n-1}{ }^{5}$. We again added block number and participants' error awareness as well as the interaction between error awareness and response-correctness $s_{n-1}$ as covariates. Our model included by-participant random slopes for ITI

\footnotetext{
${ }^{5}$ We added and tested the interaction between experiment and all factors concerning ITI but not target type as only ITI but not target type varied between experiments. To parallel our analyses of the individual experiments, we, however, added the interaction between target type and response-correctness $s_{n-1}$ as well as the covariates included in the analyses of the individual experiments.
} 
duration, response-correctness $_{n-1}$, and their interaction (note, that we tested a more parsimonious model that included only random intercepts for participants against this model and found that the more complex model fitted the data much better with a $\mathrm{BF}=39161$ in favor of the model reported here). We also estimated correlations among random effects. For regression coefficients (population-level/ fixed effects), we used moderately informative Cauchy priors with a scale of 0.353 and a mean of 0 (recently proposed default priors for logistic models; Oberauer, 2019). For random effects (standard deviations of group-level effects), we used non-negative weakly informative priors (half student t-prior with three degrees of freedom and a scaling parameter of 2.5). We used non-informative LKJ priors (shape parameter 1) for the correlations of random effects. All categorical predictors were coded as sum-to-zero contrasts and continuous predictors were mean-centered.

We estimated the posteriors by sampling parameter values using the No-U-Turn Sampler (NUTS, an extension of the Hamilton Monte Carlo sampling method) as implemented in Stan (Carpenter et al., 2017). We sampled generations through four independent Markov chains with 10,000 iterations each $(2,000$ warm up, thus a total of 32,000 post-warmup samples). To investigate convergence, we inspected Rhat values (ratio of between-chain variance to withinchain variance). Rhat values were $\leq 1.01$ for all parameters in every model. Additionally, we inspected the chains for convergence visually.

We computed Bayes Factors (BFs) to estimate the strength of evidence for the null and the alternative hypothesis. To this end, we fit two competing, nested models for each hypothesis listed above: One including the effect of interest (interaction between ITI and PIA) and one that did not include this interaction. We then compared the two models to calculate the evidence for/against the null/alternative hypothesis. Generally, a BF larger than 3 can be considered as 
substantial evidence for one hypothesis over the other (see e.g., Jarosz \& Wiley, 2014). For instance, $\mathrm{a} \mathrm{BF}_{01}$ of 3 would indicate that the data are 3 times more likely under the null hypothesis than under the alternative hypothesis and would thus demonstrate evidence against an influence of ITI on PIA effects. The goal of this pooled Bayesian analysis was to answer the question whether there was substantial evidence against an influence of ITI duration on the strength of the PIA effect. To this end, we first assessed whether the interaction between ITI

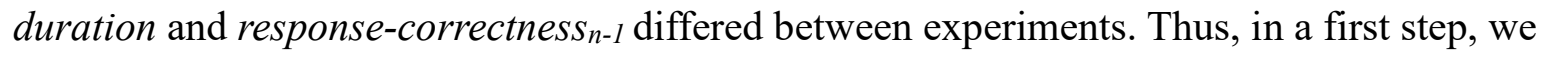
tested whether a model including the three-way interaction between ITI duration, response-correctness $s_{n-1}$, and experiment was superior to a model without that interaction. This was not the case $\left(\mathrm{BF}_{01}=8.7\right)$. Therefore, we did not further consider this three-way interaction. If evidence in favour of the three-way interaction had existed, we would have additionally accounted for differences between experiments in all following model comparisons. In a second step, we tested a model with an interaction between ITI duration and response-correctness $s_{n-1}$ against a model without that interaction. We found substantial evidence against the ITI duration and response-correctness $s_{n-1}$ interaction. The data was more likely to be observed under a model without that interaction than under a model with that interaction $\left(\mathrm{BF}_{01}=6.0\right)$.

\section{Discussion}

In Experiment 2, participants' to-be-performed response in a given trial $\mathrm{n}_{\mathrm{n}}$ again either repeated or switched as compared to the correct response of the preceding trial $\mathrm{n}_{\mathrm{n}-1}$. Again, we manipulated the preparation time after an error. This time, participants were provided with even more time for the long ITI (3000 ms as compared to $1700 \mathrm{~ms}$ in Experiment 1 ; RSI $=3800 \mathrm{~ms}$ vs. $2500 \mathrm{~ms}$ in Experiment 1). Our aim was to replicate the findings of Experiment 1 and 
demonstrate that evidence in favor of the memory obstruction hypothesis can be found even when participants are given ample preparation time.

Again, we replicated the typical PES effect for short ITIs, suggesting that our experiment led to post-error processing that was comparable to prior studies not using response repeat/switch targets (e.g., Notebaert et al., 2009; Rabbitt, 1966). Furthermore, participants again demonstrated high RAA for incorrect and correct responses, replicating prior research (e.g., Boldt \& Yeung, 2015; Grützmann et al., 2014; Klein et al., 2013; Steinhauser \& Yeung, 2010). Thus, error awareness, a pre-condition for controlled post-error adaptations, could also be confirmed for Experiment 2. Thus, again, our results cannot alternatively be explained by a lack of typical posterror processing or a lack of error awareness.

Overall, we were able to replicate the findings of Experiment 1. Crucially, even for very long ITI durations of $3000 \mathrm{~ms}(\mathrm{RSI}=3800 \mathrm{~ms})$, participants did not commit significantly fewer errors in post-error as compared to post-correct trials. In contrast to Experiment 1, we observed no inversed PIA effect for response switch targets, but for response repeat targets post-hoc comparisons suggested participants were able to improve in trials following an error on some occasion (and reactivate the current task-set related representations). Nevertheless, in line with the memory obstruction hypothesis, the interaction of target type and PIA pointed towards increased problems with reconfiguring the task-set related representations (e.g., task-set translations) for response switch as compared to response repeat targets (i.e., when resource demands were increased).

We explain the observed stronger memory obstruction for response switch as compared to response repeat targets as follows: In our design, response switch targets generally necessitate a multi-step process to determine the correct response, whereas a single step is sufficient on 
response repeat trials (see also Discussion of Experiment 1 for details). Yet, this was not the case for Experiment 1 and thus we cannot draw definitive conclusions for response repeat targets. For response switch targets however, we find very clear evidence for an inversed PIA effect in Experiment 1 and failed to find a PIA effect in Experiment 2 - both times irrespective of the ITI duration (see also pooled Bayesian analysis). We take this as evidence for the assumption that attentional resources are withdrawn from the current task-set related representation (or it is disrupted) and that even when adaptive error processing is complete, participants struggle to reactivate task-set related representations held in WM in the current paradigm.

For PES analyses, we found that PES was no longer observed for the long $3000 \mathrm{~ms}$ ITI $(\mathrm{RSI}=3800 \mathrm{~ms})$. However, post-hoc comparisons suggested that this interaction was not driven by a decrease in RTs in post-error trials from the short to the long ITI, but by an increase in RTs for post-correct trials with increasing ITI duration. Descriptively, this also matches the pattern observed in Experiment 1. The observed difference in RTs between the short and long ITIs for post-correct but virtually no difference for post-error trials is interesting. First, the observation that RTs in post-error trials do not differ between short and long ITIs contrasts with prior studies assessing the impact of ITI on PES (here, RTs in post-error decrease with increasing ITI; e.g., van der Borght et al., 2016). This might suggest that the typical PES effect does indeed not disappear but is overshadowed by another effect. Second, this other effect we observed in postcorrect trials implies that, for very long ITIs, either an additional process leading to prolonged RTs occurs or processing steps also occurring during short ITIs take longer (e.g., temporal learning, see e.g., Schmidt, 2013, affecting RTs might be less precise for longer time intervals; see e.g., Hayashi, et al. 2014, for evidence that timing abilities in the sub- and supra-second range might differ). At present, we cannot tell which processes are at the core of this finding. 
Regardless of the what the exact processes are, however, our findings suggest that these processes might also be obstructed post-error, as we do not see a corresponding RT increase with ITI duration in post-error trials. Moreover, it is crucial to note that although PES effects vanish for long ITIs in the current study, we do not find an overall PIA effect or an impact of ITI on it (see also pooled Bayesian analysis). This strongly suggests that our findings cannot alternatively be explained by an attentional bottleneck that could not be resolved in Experiment 1, but rather resulted from obstructed memory.

Taken together, our findings are again in line with a weak version of the memory obstruction hypothesis: Representations of the current task-set related representations (e.g., pastspecific task-set translations and/or past responses) are obstructed upon an error rendering a subsequent reconfiguration of the task-set difficult when response repeat/ (and especially) switch targets are used. We suggest that, as a result, controlled post-error processing cannot successfully finish and the prior task-set needs to be reinstated from partially existing memory (see General discussion). As a consequence, accuracy does not benefit in post-error trials (except when responses repeat when ample preparation time is given in one of the ITI conditions). We will discuss the difference between response repeat and switch targets and the implications of our findings for the involvement of WM and LTM in detail in the General Discussion.

\section{General Discussion}

In two experiments, we investigated how post-error cognitive control and current task-set related representations in (working) memory interacted. To do so, we used response repeat/switch targets to ensure that related task-set representations needed to be updated on a trial-by-trial basis as participants' memory of the previously given response determined their response in the following trial. Based on the memory obstruction hypothesis, we predicted that 
post-error adaptations would disrupt the task-set translation updating process in-between trials and simultaneously withdraw attention from the rather fragile, task-set related representations in WM. This would render it more difficult (weak memory obstruction hypothesis) or even impossible (strong memory obstruction hypothesis) to reactivate task-set related representations when the automatic post-error processes (e.g., attentional orienting towards the source of the error) are completed. The following controlled post-error processes, such as a successful task-set reconfiguration or retuning, depend upon task-set memory. Thus, controlled post-error processes leading to performance improvements following errors should not occur (weak memory obstruction hypothesis) or there might even be substantial performance decrements post-error (strong memory obstruction hypothesis).

\section{Manipulation Checks}

First, we needed to ensure that post-error processing in our paradigm was comparable to prior studies even though we expected results to critically diverge from prior studies in several central aspects related to controlled post-error processing. To do so, we needed to determine that post-error processing that should be unaffected by memory obstruction (PES for short ITIs; Notebaert et al., 2009; Rabbitt, 1966) in our study was comparable to prior studies. In both experiments, we found a distinct PES effect for short ITIs which corresponded to the size of PES effects reported in prior studies (e.g., Notebaert et al., 2009; Rabbitt, 1966). Thus, our results cannot alternatively be explained by assuming that participants did not engage in typical posterror processing. Second, post-error processing (e.g., neuronal error-processing markers as well as PES) has been linked to RAA (e.g., Boldt \& Yeung, 2015; Grützmann et al., 2014; Klein et al., 2013; Steinhauser \& Yeung, 2010). We therefore needed to ensure that participants were aware of the correctness of their response to a sufficient degree. RAA rates substantially above chance 
for both correct as well as erroneous responses (comparable to prior studies: see e.g., Grützmann et al., 2014; Hughes \& Yeung, 2011; Murphy et al., 2012; Shalgi et al., 2009; Shalgi \& Deouell, 2012; Wang et al., 2020, for similar results; however, note that in some studies using error signaling tasks instead of a two alternative forced choice task, error detection rates can be higher: see e.g., Maier et al., 2008) clearly indicate that this was the case. Thus, our results also cannot alternatively be explained by assuming that participants were not sufficiently aware of the correctness of their responses. Moreover, by including RAA ${ }^{6}$ in our models, we accounted for any individual differences in error awareness and their impact on post-error adaptations.

\section{Evidence in Favor of The Memory Obstruction Hypothesis}

To test the interdependence of cognitive control and memory in post-error processing, we built on concurrent theories of post-error processing (see Wessel, 2018) that suggest that following errors, the current task-set is inhibited or disrupted: In our response repeat/switch target paradigm, participants had to continuously hold task-set related representations in WM and

${ }^{6}$ Note that, we did not make assumptions about potential causes of an initial error in our study which represent an interesting research question by itself. Neverthless, we can already rule out some of these potential error causes as the basis of our findings. One could assume that errors occur due to a suboptimally represented task set (i.e., the resulting reduced error awareness), increasing the likelihood of error sequences. However, in our experiments, participants were presented with feedback regarding the correctness of their responses and had ample time to adapt. Furthmore, as stated before, error awareness was comparable to prior studies and controlled for. 
update them according to prior responses to prevail. In line with the theory of Wessel (2018), we hypothesized that task-set related representations currently held in an active state in (working) memory would be obstructed following an error and thus at best less accessible, at worst removed. Consequently, this should render controlled post-error processing that relies on task-set related representations in (working) memory ineffective. As performance in the present paradigm fully relies on WM representations of the previously given response and the updated task-set related information, we expected not to see typical post-error performance improvements even when participants were given ample preparation time.

We first argued that in the present response repeat/switch target paradigm, participants should not be able to build on either memory for the previous response or proactively-prepared task-set translations following errors and should thus show an inversed PIA effect (strong version of the memory obstruction hypothesis) or at least no PIA effect (weak version) even for long ITIs. In line with the weak version of the memory obstruction hypothesis, irrespective of the ITI duration, there was no PIA effect in Experiment 1 (long ITI duration 1700 ms/ RSI duration 2500 ms) or Experiment 2 (long ITI duration $3000 \mathrm{~ms} / \mathrm{RSI}$ duration $3800 \mathrm{~ms}$ ). This finding is in line with the weak version of the memory obstruction hypothesis as well as Wessel's (2018) adaptive orienting theory. In the most extreme case, we would even have expected an inversed PIA effect and post-error performance at chance level. Although there was evidence of an inversed PIA effect in specific conditions, our findings do not align with a strong version of the memory obstruction hypothesis. They rather point towards a weak version of the memory obstructions hypothesis: That is, task-set related representations were disrupted/inhibited, but not completely removed from WM. We will further discuss the exact connections between cognitive control and memory after elaborating on the other effects that speak to the memory obstruction hypothesis. 
Please note that Wessel (2018) proposes inhibitory/ suppressive processes in his theory. If inhibition were the mechanism underlying the observed memory obstruction (the present research remains agnostic about this question), future research will need to use very short ITIs/RSIs to elaborate on inhibitory contributions. Thereby, it would be possible to assess to what extent task-set memory is still accessible shortly after an error.

Second, because memory obstruction prevents successful adaptations following an error (i.e., controlled error processing, such as the reconfiguration or tuning of the prior task-set), PES should be prolonged and thus still be observed for longer ITIs. Again, our findings in support this notion (though additional processes seem to play a role for the long ITI condition in Experiment 2). This finding is notable different from previous studies demonstrating reduced PES effects with increasing ITIs or RSI (e.g., Buzzell et al., 2017; Jentzsch \& Dudschig, 2009; Purcell \& Kiani, 2016; Ullsperger \& Danielmeier, 2016; van der Borght et al., 2016). Our results consequently support the notion that, for response switch/repeat targets, controlled post-error adaptions are interrupted/hindered even for long ITIs and the subsequent need to draw attention back to the prior task-set (see Wessel, 2018, for how PES reflects the delay associated with a premature reorientation back towards the inital task-set) results in prolonged PES. These findings again speak towards the weak version of the memory obstruction hypothesis.

Last, the interruption of the current task-set related representations in WM should result in worse performance especially for response switch trials. That is, in post-error trials with a response switch target, participants need to not only remember and correct their previous response (e.g., left key press was incorrect; right key press would have been correct), but they also have to then devote cognitive resources to preparing the opposite response (e.g., right key press would have been correct in the previous trial; in a response switch trial, a left key press is 
demanded). As a result, participants should have greater difficulties to reorient back towards the prior task-set related representations for response switch as compared to response repeat targets especially in post-error trials as compared to post-correct trials.

The results of Experiment 1 and 2 provided evidence for this assumption. In Experiment 1, we observed an inversed PIA effect and stronger PES for response switch rather than repeat targets. In Experiment 2, again PIA effects were reduced for response switch as compared to response repeat targets. Furthermore, in both experiments, PES was again stronger for response switch as compared to response repeat targets. These findings are in line with the idea that the increased cognitive resource demands for response switch targets added to the difficulty to reconstruct the obstructed task-set related representation in (working) memory in post-error trials. Thus, our findings again provide support for the weak version of the memory obstruction hypothesis.

In addition to these arguments, we would like to highlight a possible alternative explanation for the reported influence of response target type on PIA/ PES effects based on response inhibition. After an error, a response switch target requires the same response that had been previously executed in the error trial. Wessel (2018) discusses that upon errors not only cognitive representations, but also motor representations are inhibited. Thus, the previously given response should suffer from automatic post-error control processes (i.e., inhibition) following an error. As a consequence, we should observe higher RTs in post-error trials particularly when the current trial requires the same response as the preceding error trial (i.e., when a response switch target appears post-error). Interestingly, similar mechanisms have been proposed in the task switching literature when participants switch back to a previously abandoned task (backward inhibition: impaired performance for ABA as compared to ABC task 
sequences, see e.g., Mayr \& Keele, 2000). Moreover, after a task switch, response repetitions have been found to result in slower responses and/or higher error rates and not - as normally found for task repetitions - in improved performance (e.g., Rogers \& Monsell 1995). Explaining these findings Rogers and Monsell (1995) propose that task switches are accompanied by a control mechanism, resulting in the transient suppression of all active responses. Consequently, upon a task switch, the most recent response (e.g., trial n-1 = left response) is inhibited. Thus, when the subsequent target requires the same response (left response), greater response activation is needed to overcome this inhibition (Rogers \& Monsell, 1995). Findings in the task switching literature mirror the RT results reported in the present study and inhibitory processes assumed to underly these findings in task switching (e.g., Rogers \& Monsell, 1995) mirror explanations of Wessel (2018) regarding inhibition in post-error control. These parallels open up interesting avenues for future research to compare potential inhibitory mechanism (i.e., a specific cognitive control operations) following errors and task-switches (see also Meiran, 1996, 2000, for discussion for potential inhibitory or task-set reconfiguration processes in task switching).

\section{The Role of Memory for Post-Error Control}

Yet, how exactly do post-error cognitive control processes disrupt task-set related representations (e.g., task-set translations and/or memory for the previously given response in WM) and what is the role of WM and LTM for those processes? The present study strongly suggests an interdependence of post-error cognitive control and memory. At present, it is still uncertain by what exact mechanism task-set representations in WM are disrupted. That is, we cannot yet determine with certainty whether task-set representations are removed from WM (see Lewis-Peacock et al., 2018 and Oberauer, 2018 for the idea that information can be removed from WM), inhibited (see Wessel, 2018; e.g., by a suppressive mechanism, Wessel et al., 2016), 
or disrupted in another, yet unspecified way. However, we do find evidence that task-set related representations in WM could be reinstated. Based on this finding, we elaborate in detail on how LTM and WM would have needed to interact to reinstate task-set related representations in WM given each possible mechanism of memory obstruction.

In a first scenario, participants completely removed information about the previously given response and task-set translations from WM following an error. If that were the case, they should only have been able to reinstate the task-set if all necessary information had already been encoded into LTM. It is likely that participants encoded the meaning of the response repeat/switch targets in LTM. However, it is far less likely that the just given response or already flexibly-updated task-set translations (e.g., now: if repeat target, press right, because the previous response was right) were stored in LTM with a sufficient strength. Especially, participants could only have relied on such task-set translations (proactively prepared during responding) for postcorrect trials, but not for post-error trials. On post-error trials, proactively prepared task-set translations would have been doomed to fail. To succeed in reinstating the task-set in post-error trials, participants would have had to both retrieve the proactively prepared task-set translations and initiate a negation process upon registering an error. At best, one could assume that participants only kept vowel/consonant-repeat/switch mappings in LTM. If so and if they additionally used tactile after sensations of their prior response or a response strategy like lifting the finger of the just given response, they might have reconstructed the task-set related information from scratch (as they were on average sufficiently aware of errors and also received error feedback). That is, they could have negated the previously given response to adapt the reference response for the next trial and then, by referring to the vowel/consonant-repeat/switch mappings, have repeated or switched it accordingly. Yet, participants RTs were relatively short. It 
remains questionable whether this reconstruction could have occurred this fast. If it did, we would have at least expected strong performance decrements for short ITIs. We did, however, not find evidence for an interaction between ITI and PIA effects, deeming this explanation less likely. In summary, all explanations assuming that task-set related representations were completely removed from WM postulate that multiple processes occurred in parallel or in rapid sequence to restore the task-set. This appears unparsimonious.

Alternatively, participants' task-set related representations in WM either were only partially removed, inhibited, or otherwise disrupted without fully destroying them. This second scenario bases on the assumption that task-set translations are not only proactively prepared and maintained in WM, but also weighted at least during post-error processing (though the assumed weighting mechanism might slightly differ; for the sake of simplicity we will not go into more detail in this respect; see Memelink \& Hommel, 2013, for intentional weighting as a basic principle in cognitive control; see also Hommel et al., 2014 for evidence that cognitive control might not affect the creation of bindings - here task-set related representations - but rather their retrieval). That is, post-error weighting makes current task-set related representations in WM less accessible, but these weights could subsequently be adapted again to allow a re-orienting towards the task-set and enable adaptive post-error improvements. This would imply that task-set related representations themselves remain within WM, but as they are less accessible post-error any processes using them would be less effective until the weights are re-adjusted. Regardless of how the exact process underlying this weighting compare to other mechanisms (e.g., partial removal vs. inhibition), a weighting account can explain our findings which support a weak version of the memory obstruction hypothesis. Note, that intentional weighting assumes that the weights of features that are coded on task-relevant dimensions are changed (Memelink \& Hommel, 2013): 
interrupting such a process would then also ultimately lead to the obstruction of (forming) memory. Importantly, however, such an obstruction mechanism for the task-set memory can nonetheless largely be resolved. This speaks to the adaptive nature of the interplay of cognitive control and memory.

Taken together, although our results cannot clearly favor one of the two scenarios, based on its parsimony, a weighting of task-set related representations held in WM appears more sensible to us at present. Future research needs to further dissociate the contributions of WM and LTM to post-error control and pinpoint what exact mechanisms (e.g., partial removal vs. inhibition) are causing a disruption of WM representations and/or ongoing processes building on them. Combining WM tasks with experimental designs intended to measure post-error adaptations seems promising to achieve a deeper understanding of the role of WM in post-error processing. Regardless of the results of such future studies, here, we already provide clear evidence that task-set related representations in WM are obstructed based on post-error cognitive control processes.

\section{Conclusion}

In conclusion, participants in our study showed typical post-error processing (PES for short ITIs) and error awareness. Nonetheless, our findings crucially diverge from prior studies in a number of essential aspects that support a weak version of the memory obstruction hypothesis. Thus, post-error cognitive control and memory are intertwined. After an error, cognitive control processes disrupt task-set related representations in WM and prevent post-error cognitive control processes from effectively improving performance when task-sets are flexible and (at least mainly) stored in WM. Thus, we demonstrate that cognitive control influences memory and, conversely, memory is essential for cognitive control to take effect. Using response repeat/switch 
targets, we provide a novel method of studying this interdependence of post-error cognitive control and (working) memory.

\section{References}

Baayen, R. H., Davidson, D. J., \& Bates, D. M. (2008). Mixed-effects modeling with crossed random effects for subjects and items. Journal of Memory and Language, 59(4), 390-412. https://doi.org/10.1016/j.jml.2007.12.005

Band, G. P., van Steenbergen, H., Ridderinkhof, K. R., Falkenstein, M., \& Hommel, B. (2009). Action-effect negativity: Irrelevant action effects are monitored like relevant feedback. Biological Psychology, 82(3), 211-218. https://doi.org/10.1016/j.biopsycho.2009.06.011

Barr, D. J., Levy, R., Scheepers, C., \& Tily, H. J. (2013). Random effects structure for confirmatory hypothesis testing: Keep it maximal. Journal of Memory and Language, 68(3), 255-278. https://doi.org/10.1016/j.jml.2012.11.001

Bates, D., Mächler, M., Bolker, B., \& Walker, S. (2015). Fitting Linear Mixed-Effects Models Using lme4. Journal of Statistical Software, 67(1), 1-48. https://doi.org/10.18637/jss.v067.i01

Boldt, A., \& Yeung, N. (2015). Shared Neural Markers of Decision Confidence and Error Detection. The Journal of Neuroscience, 35(8), 3478. https://doi.org/10.1523/JNEUROSCI.0797-14.2015

Bugg, J. M., \& Crump, M. J. (2012). In support of a distinction between voluntary and stimulusdriven control: A review of the literature on proportion congruent effects. Frontiers in Psychology, 3, 367. https://doi.org/10.3389/fpsyg.2012.00367

Bürkner, P.-C. (2017). brms: An R package for Bayesian multilevel models using Stan. Journal of Statistical Software, 80(1), 1-28. 
Bürkner, P.-C. (2018). Advanced Bayesian multilevel modeling with the R package brms. $R$ Journal, 10(1), 395-411. https://doi.org/10.32614/rj-2018-017

Buzzell, G. A., Beatty, P. J., Paquette, N. A., Roberts, D. M., \& McDonald, C. G. (2017). ErrorInduced Blindness: Error Detection Leads to Impaired Sensory Processing and Lower Accuracy at Short Response-Stimulus Intervals. The Journal of Neuroscience: The Official Journal of the Society for Neuroscience, 37(11), 2895-2903. https://doi.org/10.1523/JNEUROSCI.1202-16.2017

Carpenter, B., Gelman, A., Hoffman, M. D., Lee, D., Goodrich, B., Betancourt, M., ... \& Riddell, A. (2017). Stan: a probabilistic programming language. Grantee Submission, 76(1), 1-32.

Cortis, C., Dent, K., Kennett, S., \& Ward, G. (2015). First things first: Similar list length and output order effects for verbal and nonverbal stimuli. Journal of Experimental Psychology: Learning, Memory, and Cognition, 41(4). https://doi.org/10.1037/xlm0000086

Cowan, N. (1999). An embedded-processes model of working memory.

In A. Miyake \& P. Shah (Eds.), Models of working memory: Mechanisms of active maintenance and executive control (pp. 62-101). Cambridge, UK: Cambridge University Press. https://doi.org/10.1017/CBO9781139174909.006

Crump, M. J., \& Logan, G. D. (2010). Contextual control over task-set retrieval. Attention, Perception, \& Psychophysics, 72(8), 2047-2053. https://doi.org/10.3758/BF03196681

Danielmeier, C., \& Ullsperger, M. (2011). Post-Error Adjustments. Frontiers in Psychology, 2, 233. https://doi.org/10.3389/fpsyg.2011.00233

Di Gregorio, F., Maier, M. E., \& Steinhauser, M. (2018). Errors can elicit an error positivity in the absence of an error negativity: Evidence for independent systems of human error monitoring. Neuroimage, 172, 427-436. https://doi.org/10.1016/j.neuroimage.2018.01.081 
Dreisbach, G. (2012). Mechanisms of cognitive control: The functional role of task rules. Current Directions in Psychological Science, 21(4), 227-231. https://doi.org/10.1177/0963721412449830

Dutilh, G., van Ravenzwaaij, D., Nieuwenhuis, S., van der Maas, H. L., Forstmann, B. U., \& Wagenmakers, E.-J. (2012). How to measure post-error slowing: A confound and a simple solution. Journal of Mathematical Psychology, 56(3), 208-216. https://doi.org/10.1016/j.jmp.2012.04.001

Egner, T. (2008). Multiple conflict-driven control mechanisms in the human brain. Trends in Cognitive Sciences, 12(10), 374-380. https://doi.org/10.1016/j.tics.2008.07.001

Falkenstein, M., Hohnsbein, J., \& Hoormann, J. (1993). Late visual and auditory ERP components and choice reaction time. Biological Psychology, 35(3), 201-224. https://doi.org/10.1016/0301-0511(93)90002-P

Gehring, W. J., Goss, B., Coles, M. G. H., Meyer, D. E., \& Donchin, E. (1993). A Neural System for Error Detection and Compensation. Psychological Science, 4(6), 385-390. https://doi.org/10.1111/j.1467-9280.1993.tb00586.x

Grützmann, R., Endrass, T., Klawohn, J., \& Kathmann, N. (2014). Response accuracy rating modulates ERN and Pe amplitudes. Biological Psychology, 96, 1-7. https://doi.org/10.1016/j.biopsycho.2013.10.007

Hayashi, M. J., Kantele, M., Walsh, V., Carlson, S., \& Kanai, R. (2014). Dissociable neuroanatomical correlates of subsecond and suprasecond time perception. Journal of Cognitive Neuroscience, 26, 1685-1693. https://doi.org/10.1162/jocn_a_00580

Hauser, T. U., Iannaccone, R., Stämpfli, P., Drechsler, R., Brandeis, D., Walitza, S., \& Brem, S. (2014). The feedback-related negativity (FRN) revisited: New insights into the localization, 
meaning and network organization. NeuroImage, 84, 159-168.

https://doi.org/10.1016/j.neuroimage.2013.08.028

Holroyd, C. B., \& Coles, M. G. H. (2002). The neural basis of human error processing:

Reinforcement learning, dopamine, and the error-related negativity. Psychological Review, 109(4), 679-709. https://doi.org/10.1037/0033-295X.109.4.679.

Hommel, B., Memelink, J., Zmigrod, S., \& Colzato, L. S. (2014). Attentional control of the creation and retrieval of stimulus-response bindings. Psychological Research, 78(4), 520-538. https://doi.org/10.1007/s00426-013-0503-y

Hughes, G., \& Yeung, N. (2011). Dissociable correlates of response conflict and error awareness in error-related brain activity. Neuropsychologia, 49(3), 405-415.

Jentzsch, I., \& Dudschig, C. (2009). Why do we slow down after an error? Mechanisms underlying the effects of posterror slowing. Quarterly Journal of Experimental Psychology (2006), 62(2), 209-218. https://doi.org/10.1080/17470210802240655

Judd, C. M., Westfall, J, \& Kenny, D. A. (2012). Treating stimuli as a random factor in social psychology: A new and comprehensive solution to a pervasive but largely ignored problem. Journal of Personality and Social Psychology, 103(1), 54-69. https://doi.org/10.1037/a0028347

Kenward, M. G., \& Roger, J. H. (1997). Small Sample Inference for Fixed Effects from Restricted Maximum Likelihood. Biometrics, 53(3), 983-997. https://doi.org/10.2307/2533558

Kiesel, A., Steinhauser, M., Wendt, M., Falkenstein, M., Jost, K., Philipp, A. M., \& Koch, I. (2010). Control and interference in task switching-A review. Psychological Bulletin, 136(5). https://doi.org/10.1037/a0019842 
Kikumoto, A., \& Mayr, U. (2017). The Nature of Task Set Representations in Working Memory. Journal of Cognitive Neuroscience, 29(11), 1950-1961. https://doi.org/10.1162/jocn_a_01173

Klein, T. A., Ullsperger, M., \& Danielmeier, C. (2013). Assessing error awareness without relying on introspective judgment? Frontiers in Neuroscience, 7, 113. https://doi.org/10.3389/fnins.2013.00113

Lenth, R., Singmann, H, Love, J., Buerkner, P., \& Herve, M. (2018). Emmeans: Estimated Marginal Means, AKA Least-squares Means. R Package Version 1.1. 3; 2018.

Lewis-Peacock, J. A., Kessler, Y., \& Oberauer, K. (2018). The removal of information from working memory. Annals of the New York Academy of Sciences, 1424(1), 33-44. https://doi.org/10.1111/nyas. 13714

Lüdecke, D. (2018). sjPlot: Data visualization for statistics in social science. $R$ Package Version, 2(1).

Maier, M. E., Yeung, N., \& Steinhauser, M. (2011). Error-related brain activity and adjustments of selective attention following errors. NeuroImage, 56(4), 2339-2347. https://doi.org/10.1016/j.neuroimage.2011.03.083

Marco-Pallares, J., Camara, E., Munte, T. F., and Rodriguez-Fornells, A. (2008). Neural mechanisms underlying adaptive actions after slips. Journal of Cognitive Neuroscience. 20, 1595-1610. https://doi.org/10.1162/jocn.2008.20117

Masina, F., Vallesi, A., Di Rosa, E., Semenzato, L., \& Mapelli, D. (2018). Possible Role of Dorsolateral Prefrontal Cortex in Error Awareness: Single-Pulse TMS Evidence. Frontiers in Neuroscience, 12, 179. https://doi.org/10.3389/fnins.2018.00179

Mayr, U., Kuhns, D., \& Hubbard, J. (2014). Long-term memory and the control of attentional control. Cognitive Psychology, 72, 1-26. https://doi.org/10.1016/j.cogpsych.2014.02.001 
Mayr, U., \& Keele, S. W. (2000). Changing internal constraints on action: The role of backward inhibition. Journal of Experimental Psychology: General, 129(1), 426. https://doi.org/10.1037/0096-3445.129.1.4

Meiran, N. (1996). Reconfiguration of processing mode prior to task performance. Journal of Experimental Psychology: Learning, Memory, and Cognition, 22(6), 14231442. https://doi.org/10.1037/0278-7393.22.6.1423

Meiran, N. (2000). Modeling cognitive control in task-switching. Psychological Research, 63(3), 234-249. https://doi.org/10.1007/s004269900004

Memelink, J., \& Hommel, B. (2013). Intentional weighting: A basic principle in cognitive control. Psychological Research, 77(3), 249-259. https://doi.org/10.1007/s00426-012-0435-y

Morey, R. D. (2008). Confidence intervals from normalized data: A correction to Cousineau (2005). Reason, 4(2), 61-64.

Murphy, P. R., Robertson, I. H., Allen, D., Hester, R., \& O'Connell, R. G. (2012). An electrophysiological signal that precisely tracks the emergence of error awareness. Frontiers in Human Neuroscience, 6, 65.

Oberauer, K. (2019). Working Memory Capacity Limits Memory for Bindings. Journal of Cognition, 2(1), 40. DOI: http://doi.org/10.5334/joc.86

Nakagawa, S., Johnson, P. C. d., \& Schielzeth, H. (2017). The coefficient of determination R2 and intra-class correlation coefficient from generalized linear mixed-effects models revisited and expanded. Journal of the Royal Society Interface, 14(134), 20170213. https://doi.org/10.1098/rsif.2017.0213

Nieuwenhuis, S., Ridderinkhof, K. R., Blom, J., Band, G. P., \& Kok, A. (2001). Error-related brain potentials are differentially related to awareness of response errors: Evidence from an 
antisaccade task. Psychophysiology, 38(5), 752-760. https://doi.org/10.1111/1469-

8986.3850752

Notebaert, W., Houtman, F., van Opstal, F., Gevers, W., Fias, W., \& Verguts, T. (2009). Post-error slowing: An orienting account. Cognition, 111(2), 275-279.

https://doi.org/10.1016/j.cognition.2009.02.002

Oberauer, K. (2018). Removal of irrelevant information from working memory: Sometimes fast, sometimes slow, and sometimes not at all. Annals of the New York Academy of Sciences, 1424(1), 239-255. https://doi.org/10.1111/nyas.13603

Oberauer, K. (2002). Access to information in working memory: Exploring the focus of attention. Journal of Experimental Psychology: Learning, Memory, and Cognition, 28, 411421. https://doi.org/10.1037/0278-7393.28.3.411

Oberauer, K. (2009). Design for a working memory. Psychology of Learning and Motivation, 51, 45-100. https://doi.org/10.1016/S0079-7421(09)51002-X

Oberauer, K., Lewandowsky, S., Awh, E., Brown, G. D. A., Conway, A., Cowan, N., ... Ward, G. (2018). Benchmarks for models of short-term and working memory. Psychological Bulletin, 144(9). https://doi.org/10.1037/bul0000153

Purcell, B. A., \& Kiani, R. (2016). Neural Mechanisms of Post-error Adjustments of Decision Policy in Parietal Cortex. Neuron, 89(3), 658-671. https://doi.org/10.1016/j.neuron.2015.12.027

Rabbitt, P. M. (1966). Errors and error correction in choice-response tasks. Journal of Experimental Psychology, 71(2), 264-272. https://doi.org/10.1037/h0022853 
Rogers, R. D., \& Monsell, S. (1995). Costs of a predictible switch between simple cognitive tasks. Journal of Experimental Psychology: General, 124(2), 207-

231. https://doi.org/10.1037/0096-3445.124.2.207

Schmidt, J. R. (2013). Temporal learning and list-level proportion congruency: Conflict adaptation or learning when to respond?. PLoS One, 8(11), e82320.

https://doi.org/10.1371/journal.pone.0082320

Schroder, H. S., Nickels, S., Cardenas, E., Breiger, M., Perlo, S., \& Pizzagalli, D. A. (2020). Optimizing assessments of post-error slowing: A neurobehavioral investigation of a flanker task. Psychophysiology, 57(2), e13473. https://doi.org/10.1111/psyp.13473

Shalgi, S., Barkan, I., \& Deouell, L. Y. (2009). On the positive side of error processing: errorawareness positivity revisited. European Journal of Neuroscience, 29(7), 1522-1532.

Shalgi, S., \& Deouell, L. Y. (2012). Is any awareness necessary for an Ne?. Frontiers in Human Neuroscience, 6, 124.

Singmann, H., Bolker, B., Westfall, J [Jake], \& Aust, F. (2015). afex: Analysis of factorial experiments. R Package Version 0.13-145.

Steinhauser, M., \& Yeung, N. (2010). Decision Processes in Human Performance Monitoring. The Journal of Neuroscience, 30(46), 15643. https://doi.org/10.1523/JNEUROSCI.189910.2010

Szumilas, M. (2010). Explaining odds ratios. Journal of the Canadian Academy of Child and Adolescent Psychiatry = Journal De L'academie Canadienne De Psychiatrie De L'enfant Et De L'adolescent, 19(3), 227-229. https://www.ncbi.nlm.nih.gov/pubmed/20842279

Ullsperger, M., \& Danielmeier, C. (2016). Reducing Speed and Sight: How Adaptive Is PostError Slowing? Neuron, 89(3), 430-432. https://doi.org/10.1016/j.neuron.2016.01.035 
van der Borght, L., Braem, S., Stevens, M., \& Notebaert, W. (2016). Keep calm and be patient: The influence of anxiety and time on post-error adaptations. Acta Psychologica, 164, 34-38. https://doi.org/10.1016/j.actpsy.2015.12.007

Waszak, F., Hommel, B., \& Allport, A. (2003). Task-switching and long-term priming: Role of episodic stimulus-task bindings in task-shift costs. Cognitive Psychology, 46(4), 361-413. https://doi.org/10.1016/S0010-0285(02)00520-0

Wessel, J. R. (2011). Error awareness and the error-related negativity: Evaluating the first decade of evidence. Frontiers in Human Neuroscience, 23(10), 3021-3036. https://doi.org/10.3389/fnhum.2012.00088

Wessel, J. R. (2018). An adaptive orienting theory of error processing. Psychophysiology, 55(3), e13041. https://doi.org/10.1111/psyp.13041

Wessel, J. R., Jenkinson, N., Brittain, J.-S., Voets, Sarah H. E. M., Aziz, T. Z., \& Aron, A. R. (2016). Surprise disrupts cognition via a fronto-basal ganglia suppressive mechanism. Nature Communications, 7(1), 11195. https://doi.org/10.1038/ncomms11195

Whitehead, P. S., \& Egner, T. (2018). Cognitive control over prospective task-set interference. Journal of Experimental Psychology: Human Perception and Performance, 44(5), 741-755. https://doi.org/10.1037/xhp0000493

Woods, D. L., Wyma, J. M., Herron, T. J., \& Yund, E. W. (2016). An improved spatial span test of visuospatial memory. Memory, 24(8), 1142-1155. 